SHEP 95/42

KANAZAWA 96-02

HUB-EP-96/6

HLRZ $77 / 95$

WUB 95-45

\title{
Dual Superconductor Scenario of Confinement: A Systematic Study of Gribov Copy Effects
}

\author{
G.S. Bali \\ Physics Department, The University, Highfield, Southampton SO17 1BJ, UK \\ V. Bornyakov \\ Department of Physics, Kanazawa University, Kanazawa 920-11, Japan \\ and Institute for High Energy Physics, 142284 Protvino, Russid円 \\ M. Müller-Preussker \\ Institut für Physik, Humboldt Universität zu Berlin, 10099 Berlin, Germany \\ K. Schilling \\ Fachbereich Physik, Bergische Universität, Gesamthochschule, 42097 Wuppertal, Germany \\ and HLRZ c/o KFA, 52425 Jülich, and DESY, 22603 Hamburg, Germany
}

\begin{abstract}
We perform a study of the effects from maximal abelian gauge Gribov copies in the context of the dual superconductor scenario of confinement, on the basis of a novel approach for estimation of systematic uncertainties from incomplete gauge fixing. We present numerical results, in $S U(2)$ lattice gauge theory, using the overrelaxed simulated annealing gauge fixing algorithm. We find abelian and non-abelian string tensions to differ significantly, their ratio being $0.92(4)$ at $\beta=2.5115$. An approximate factorization of the abelian potential into monopole and photon contributions has been confirmed, the former giving rise to the abelian string tension.
\end{abstract}

\footnotetext{
${ }^{1}$ Permanent address.
} 


\section{Introduction}

Understanding how confinement arises from QCD is a central problem of strong interaction physics. G. 't Hooft and S. Mandelstam proposed the QCD vacuum state to behave like a magnetic superconductor [1, 2]. A dual Meissner effect is believed to be responsible for the formation of thin string-like chromo-electric flux tubes between quarks in $S U(N)$ Yang-Mills theories. This confinement mechanism has indeed been established in compact QED [3, 4, 5]. The disorder of the related topological objects - magnetic monopoles gives rise to an area law for large Wilson loops and, thus, leads to a confining potential. The application of this idea to non-abelian gauge theories is based on the abelian projection [6], reducing the non-abelian $S U(N)$ gauge symmetry to the maximal abelian (Cartan) subgroup $U(1)^{N-1}$ by gauge fixing the off-diagonal components of the gauge field. Then the theory can be regarded as an abelian gauge theory with magnetic monopoles and charged matter fields (quarks and off-diagonal gluons). The dual superconductor idea is realized if these abelian monopoles condense. In this scenario large distance (low momentum) properties of QCD are carried by the abelian degrees of freedom (abelian dominance).

Nonperturbative investigations of this conjecture became possible after formulating the abelian projection for lattice gauge theories [7]. In previous numerical studies (see reviews [8, 9] and references therein), it has been demonstrated that the maximal abelian (MA) gauge was a very suitable candidate for lattice investigations of the 't HooftMandelstam confinement scenario, being the only known renormalizable abelian gauge.

These studies provided strong evidence for the QCD vacuum indeed to act like a dual superconductor. In Ref. [10] the value of the abelian string tension, i.e. the slope of the static potential between charge one static sources at large distance, has been observed to be close to that of the potential between static quarks in the fundamental representation of the non-abelian theory. This feature supports abelian dominance as predicted by 't Hooft. Recently, various results in favor of this picture have been obtained by other groups as well [9].

At this stage it appears to be important to address such issues on a more quantitative level. This requires a careful study of the problem of gauge (or Gribov) ambiguities [1], and the resulting biases on abelian observables. In the present paper we aim at removing this uncertainty of previous studies. We shall develop a new effective gauge fixing algorithm, thus reducing gauge fixing ambiguities with respect to the standard relaxation algorithm employed so far. We propose a numerical procedure to estimate the remaining gauge fixing biases. This enables us to carry out high precision measurements of the abelian string tension and other abelian observables, with control over systematic errors. The main results have been obtained on a $32^{4}$ lattice at $\beta=2.5115$. 
Our investigation revealed that, compared to typical statistical errors, the effect of gauge copies cannot be neglected, even with the improved gauge fixing algorithm. Nevertheless, the new algorithm does reduce the variance of observables with respect to various gauge copies considerably, compared with the traditional relaxation plus overrelaxation algorithm, and, in average, yields larger values of the functional to be maximized.

Our paper is organized as follows. In Section 2 we shortly review the status of gauge fixing and monopole kinematics to set the stage and to present the underlying formulae in a self contained fashion. In Section 3, we present the overrelaxed simulated annealing gauge fixing algorithm and compare it to the standard algorithm. In Section 4 we elaborate on a procedure to estimate the effect of remaining gauge fixing ambiguities on abelian observables, such as the monopole density or the abelian potential. Physical results on the non-abelian and abelian potentials, the decomposition of the potential into monopole and photon contributions, the abelian potential between charge two static sources and the monopole density are presented in Section 5 .

\section{Physics from maximal abelian gauge}

\subsection{Abelian projection}

We start from the lattice version [7] of 't Hooft's abelian projection [6]. The idea is to partially fix gauge degrees of freedom such that the maximal abelian (Cartan) subgroup $\left(U^{N-1}(1)\right.$ for $S U(N)$ gauge groups) remains unbroken.

A few abelian gauges have been suggested in Ref. [6] where MA gauge, referring to a differential gauge condition, has been favored. Lattice simulations have indeed demonstrated MA gauge to be very suitable for investigations of abelian projections of gauge theories.

In the following, we will restrict ourselves to the case of $S U(2)$ gauge theory. Fixing MA gauge on the lattice amounts to maximizing the functional $\left(V=N_{\text {sites }}\right)$,

$$
F(U)=\frac{1}{8 V} \sum_{n, \mu} \operatorname{Tr}\left(\sigma_{3} U_{n, \mu} \sigma_{3} U_{n, \mu}^{\dagger}\right)
$$

with respect to local gauge transformations,

$$
U_{n, \mu} \rightarrow U_{n, \mu}^{g}=g_{n} U_{n, \mu} g_{n+\hat{\mu}}^{\dagger}
$$

Condition Eq. (11) fixes (besides other possible degeneracies) $g_{n}$ only up to multiplications $g_{n} \rightarrow v_{n} g_{n}$ with $v_{n}=\exp \left(i \alpha_{n} \tau_{3}\right), \tau_{3}=\sigma_{3} / 2,-2 \pi \leq \alpha_{n}<2 \pi$, i.e. $g_{n} \in S U(2) / U(1)$. 
It has been shown that the corresponding continuum gauge, defined by the bilinear differential gauge condition,

$$
\left(\partial_{\mu} \pm i g A_{\mu}^{3}\right) A_{\mu}^{ \pm}=0 \quad, \quad A_{\mu}^{ \pm}=A_{\mu}^{1} \pm i A_{\mu}^{2} \quad,
$$

is renormalizable $[12$, a feature, that is crucial for a continuum interpretation of lattice results.

After a configuration has been transformed to satisfy the MA gauge condition, a coset decomposition is performed,

$$
U_{n, \mu}=C_{n, \mu} V_{n, \mu}
$$

where $V_{n, \mu}=\exp \left(i \phi_{n, \mu} \tau_{3}\right), \quad-2 \pi \leq \phi_{n, \mu}<2 \pi$, transforms like a (neutral) gauge field and $C_{n, \mu}$ like a charged matter field with respect to transformations within the residual abelian subgroup,

$$
V_{n, \mu} \rightarrow v_{n} V_{n, \mu} v_{n+\hat{\mu}}^{\dagger} \quad, \quad C_{n, \mu} \rightarrow v_{n} C_{n, \mu} v_{n}^{\dagger}
$$

Quark fields are also charged with respect to such $U(1)$ transformations. The abelian lattice gauge fields $V_{n, \mu}$ constitute an abelian projected configuration.

The $S U(2)$ action of the original gauge theory can be decomposed into a $U(1)$ pure gauge action, a term describing interactions of the $U(1)$ gauge fields with charged fields, i.e. the off-diagonal components, and a self-interaction term of those charged fields [13]. Maximizing the diagonal components of all gauge fields with respect to the off-diagonal components amounts to enhancing the effect of the pure $U(1)$ gauge part in comparison with those contributions containing interactions with charged fields. On a heuristic level, one might expect the importance of the $U(1)$ degrees of freedom to be enhanced in the MA projected theory, in comparison with other abelian projections.

The abelian Wilson loop for charge one static sources is defined as

$$
W^{a b}(C)=\frac{1}{2} \operatorname{Tr}\left(\prod_{l \in C} V_{l}\right)=\operatorname{Re}\left(\prod_{l \in C} u_{l}\right),
$$

where $u_{n, \mu}=\exp \left(i \theta_{n, \mu}\right), \theta_{n, \mu}=\frac{1}{2} \phi_{n, \mu}$. $C$ denotes a closed contour.

In what follows, we will use $\theta_{n, \mu}$ to specify abelian lattice fields for the sake of convenience. The "Re" symbol can be omitted from the definition of the abelian Wilson loop since expectation values of this operator become automatically real due to charge invariance $\left(\operatorname{Im}\left\langle\prod_{l \in C} u_{l}\right\rangle=0\right)$.

\subsection{Monopoles on the lattice}

One defines magnetic monopoles with respect to the residual $U(1)$ gauge group in the way proposed in Ref. [14] for $U(1)$ lattice gauge theory: abelian plaquette variables, 


$$
\theta_{n, \mu \nu}=\theta_{n, \mu}+\theta_{n+\hat{\mu}, \nu}-\theta_{n+\hat{\nu}, \mu}-\theta_{n, \nu} \quad, \quad \theta_{n, \mu \nu} \in[-4 \pi, 4 \pi)
$$

can be decomposed into a periodic (regular) part, $-\pi \leq \bar{\theta}_{n, \mu \nu}<\pi$, and a singular part, $m_{n, \mu \nu}=0, \pm 1, \pm 2$

$$
\theta_{n, \mu \nu}=\bar{\theta}_{n, \mu \nu}+2 \pi m_{n, \mu \nu} .
$$

$\bar{\theta}_{n, \mu \nu}$ describes the $U(1)$-invariant "electromagnetic" flux through the plaquette and $m_{n, \mu \nu}$ is the number of Dirac strings passing through it. Magnetic monopole currents $k_{n, \mu}$, residing on the links of the dual lattice, are defined as

$$
k_{n, \mu}=\frac{1}{4 \pi} \varepsilon_{\mu \nu \rho \sigma} \partial_{\nu} \bar{\theta}_{n, \rho \sigma}
$$

where a lattice forward derivative is used, $\partial_{\nu} f_{n}=f_{n+\hat{\nu}}-f_{n}$. It is obvious, that $k_{n, \mu}$ represents a conserved current:

$$
\sum_{\mu} \partial_{\mu} k_{n, \mu}=0
$$

Note, that the shifted currents $j_{n, \mu}=k_{n+\hat{\mu}, \mu}$ form closed loops on the dual lattice. As it has already been mentioned, first lattice results on abelian dominance have been obtained in Ref. [10] where the string tension computed from abelian Wilson loops after MA projection, Eq. (6), has been numerically found to agree approximately with the string tension as extracted from the full, non-abelian theory. Since then additional lattice results in support of the 't Hooft-Mandelstam confinement scenario have been found [8, 9]. In particular the monopole contribution to the abelian string tension has been investigated.

\subsection{Photon and monopole dynamics}

It is known that in the Villain formulation of compact $U(1)$ lattice gauge theory one meets an exact factorization of expectation values of Wilson loops into monopole and photon parts [4] and one would expect to encounter remnants of this in other lattice formulations of the theory, in the form

$$
\left\langle W^{U(1)}\right\rangle \approx\left\langle W^{m o n}\right\rangle\left\langle W^{p h}\right\rangle
$$

This factorization would induce a decomposition of the potential between static charges at separation $\mathbf{R}$,

$$
V^{U(1)}(\mathbf{R}) \approx V^{m o n}(\mathbf{R})+V^{p h}(\mathbf{R})
$$

where the photon contribution is expected to be Coulomb-like.

The decomposition rule, Eq. (12), has indeed been verified to hold approximately in simulations [15] for the case of compact $U(1)$ with Wilson action. Similarly, in MA 
projected $S U(2)$ gauge theory, abelian Wilson loop potentials were found to decompose qualitatively 16, 17].

As we aim at more quantitative conclusions about the rôle of monopoles and photons within gluodynamics we will proceed to collect all necessary formulae, following essentially Ref. [5].

The abelian Wilson loop operator Eq. (6) can be easily transformed to

$$
W^{a b}(C)=\exp \left(-\frac{i}{2} \sum_{n, \mu \nu} \bar{\theta}_{n, \mu \nu} M_{n, \mu \nu}\right) \quad,
$$

where $M_{n, \mu \nu}$ is an integer valued antisymmetric field, living on plaquettes, satisfying the condition, $\partial_{\mu}^{-} M_{n, \mu \nu}=J_{n, \nu} ; J_{n, \nu}$ being an external current associated to the (oriented) contour $C$ where it takes the values $\pm 1 . \partial_{\mu}^{-}$denotes the lattice backward derivative, $\partial_{\mu}^{-} f_{n}=f_{n}-f_{n-\hat{\mu}}$. Let us now rewrite $\bar{\theta}_{n, \mu \nu}$ in terms of dual potentials $\rho_{n, \mu}$, and a photon field $\theta_{n, \mu}^{\prime}$,

$$
\bar{\theta}_{n, \mu \nu}=\epsilon_{\mu \nu \alpha \beta} \partial_{\alpha}^{-} \rho_{n, \beta}+\partial_{\mu} \theta_{n, \nu}^{\prime}-\partial_{\nu} \theta_{n, \mu}^{\prime}+\bar{\theta}_{\mu \nu}^{0},
$$

where $\bar{\theta}_{\mu \nu}^{0}$ are zero modes defined by

$$
\bar{\theta}_{\mu \nu}^{0}=\frac{1}{V} \sum_{n} \bar{\theta}_{n, \mu \nu}=-\frac{2 \pi}{V} \sum_{n} m_{n, \mu \nu} .
$$

The dual vector potential satisfies the equation,

$$
\partial_{\nu} \partial_{\nu}^{-} \rho_{n, \mu}-\partial_{\mu}^{-} \partial_{\nu} \rho_{n, \nu}=-2 \pi k_{n, \mu}
$$

By imposing the Lorentz gauge condition $\left(\partial_{\mu} \rho_{n, \mu}=0\right)$ one finds,

$$
\rho_{n, \mu}=2 \pi \sum_{m} D(n-m) k_{m, \mu}+\text { const. , }
$$

where $D(n-m)$ denotes the lattice Coulomb propagator. Eqs. (14), (15) and (17) define $\theta_{n, \mu}^{\prime}$ up to an irrelevant constant. By inserting Eqs. (14) and (17) into Eq. (13), one arrives at

$$
W^{a b}=W^{m o n} W^{p h} W^{f v},
$$

where

$$
\begin{gathered}
W^{m o n}=\exp \left(-2 \pi i \sum_{n, m} K_{n, \mu} D(n-m) k_{m, \mu}\right) \quad, \quad K_{n, \mu}=\frac{1}{2} \epsilon_{\nu \mu \alpha \beta} \partial_{\nu} M_{n, \alpha \beta} \\
W^{p h}=\exp \left(-i \sum_{n} \partial_{\mu} \theta_{n, \nu}^{\prime} M_{n, \mu \nu}\right)=\exp \left(i \sum_{n} \theta_{n, \nu}^{\prime} J_{n, \nu}\right)
\end{gathered}
$$




$$
W^{f v}=\exp \left(-\frac{i}{2} \bar{\theta}_{\mu \nu}^{0} \sum_{n} M_{n, \mu \nu}\right),
$$

where the latter expression denotes a finite volume contribution. After applying Eq. (14), $W^{p h}$ can be written in a form, that is more convenient for calculations,

$$
W^{p h}=\exp \left(-i \sum_{n, m} \partial_{\mu}^{-} \bar{\theta}_{n, \mu \nu} D(n-m) J_{m, \nu}\right) \quad .
$$

It is worth mentioning that in Refs. [15, 16, 17] only monopole and photon contributions have been considered while the finite volume contribution has been neglected. As pointed out above, for $U(1)$ gauge theory with Villain action, it can be shown that Eq. (18) holds for expectation values of Wilson loops as well.

It is straightforward to generalize Eqs. (19) - (21) to the case of extended monopoles [18] of size $l$. These are defined as

$$
k_{n, \mu}^{(l)}=\frac{1}{4 \pi} \varepsilon_{\mu \nu \rho \sigma} \partial_{\nu}^{(l)} \bar{\theta}_{n, \rho \sigma}^{(l)}=\sum_{m \in c_{l}} k_{m, \mu}
$$

where $\partial_{\nu}^{(l)} f_{n}=f_{n+l \hat{\nu}}-f_{n}, \bar{\theta}_{n, \mu \nu}^{(l)}=\sum_{m \in s_{l}} \bar{\theta}_{n, \mu \nu}, c_{l}$ and $s_{l}$ are cubes and squares of linear extent $l$. Subsequently, the operator $W^{a b}(C)$ can be decomposed in the same way as in Eq. (18) with the monopole contribution defined by

$$
W^{(l), m o n}=\exp \left(-2 \pi i \sum_{n, m} K_{n, \mu}^{(l)} D^{(l)}(n-m) k_{m, \mu}^{(l)}\right) \quad, \quad K_{n, \mu}^{(l)}=\frac{1}{2} \epsilon_{\nu \mu \alpha \beta} \partial_{\nu}^{(l)} M_{n, \alpha \beta}
$$

$D^{(l)}(n-m)$ being the lattice Coulomb propagator on a lattice of linear dimension $L / l$.

It is useful to apply an alternative decomposition of the abelian Wilson loop into monopole and photon parts, based on the monopole vector potential [5], $\theta_{n, \mu}^{\text {mon }}=\theta_{n, \mu}-\theta_{n, \mu}^{\prime}$. From Eqs. (8) and (14) one finds $\theta_{n, \mu}^{\text {mon }}$ to satisfy the condition

$$
\partial_{\nu} \partial_{\nu}^{-} \theta_{n, \mu}^{m o n}-\partial_{\nu} \partial_{\mu}^{-} \theta_{n, \nu}^{m o n}=2 \pi \partial_{\rho}^{-} m_{n, \rho \mu} \quad,
$$

which has the Lorentz gauge solution,

$$
\theta_{n, \mu}^{m o n}=-2 \pi \sum_{m} D(n-m) \partial_{\nu}^{-} m_{m, \nu \mu}+\text { const. . }
$$

We can now write

$$
W^{a b}(C)=\exp \left(i \sum_{l \in C} \theta_{l}^{m o n} J_{l}\right) \exp \left(i \sum_{l \in C} \theta_{l}^{\prime} J_{l}\right) \equiv W^{m o n, f v}(C) W^{p h}(C)
$$


In Eq. (27), $W^{m o n, f v}$ combines both, monopole and finite volume contributions. This representation has the advantage that — provided the vector potentials $\theta_{n, \mu}^{\text {mon }}$ and $\theta_{n, \mu}^{\prime}$ have been computed - smearing techniques [19] can be applied, which have been proven to be very useful in extracting potentials from Wilson loops.

\subsection{Charge two potential}

From Eq. (4), the following decomposition of link variables within the adjoint representation $U_{n, \mu}^{A}$ can be derived:

$$
U_{n, \mu}^{A}=C_{n, \mu}^{A} V_{n, \mu}^{A}
$$

where

$$
\left(C_{n, \mu}^{A}\right)_{\alpha \beta}=\frac{1}{2} \operatorname{Tr}\left[\sigma_{\alpha} C_{n, \mu} \sigma_{\beta} C_{n, \mu}^{\dagger}\right]
$$

and

$$
\left(V_{n, \mu}^{A}\right)_{\alpha \beta}=\frac{1}{2} \operatorname{Tr}\left[\sigma_{\alpha} V_{n, \mu} \sigma_{\beta} V_{n, \mu}^{\dagger}\right]=\exp \left(i T_{3} \phi_{n, \mu}\right) \quad .
$$

$T_{3}$ is the generator of the adjoint representation and the link angle $\phi_{n, \mu}$ has been defined above.

The "adjoint" abelian Wilson loop,

$$
W^{a b, a d j}(C)=\frac{1}{3} \operatorname{Tr}_{A}\left[\prod_{l \in C} V_{l}^{A}\right],
$$

can be expressed in terms of abelian link angles

$$
W^{a b, a d j}(C)=\frac{1}{3}\left(1+2 \cdot W^{a b, 2}(C)\right),
$$

with

$$
W^{a b, 2}(C)=\cos \left(2 \theta_{C}\right) \quad, \quad \theta_{C}=\sum_{l \in C} \theta_{l} .
$$

The adjoint static "quark" field has one neutral and two charged components with respect to residual abelian gauge transformations. The neutral component does not interact with abelian gauge fields and gives rise to the constant on the right hand side of Eq. (32). Thus, it is evident that, for a full description of the interaction between adjoint sources, effects from the off-diagonal gluon fields $C_{n, \mu}^{a d j}$ have to be considered. However, one still might expect abelian dominance to hold for charged quarks. We define the abelian projected analogue of the adjoint potential, $V^{a b, a d j}(R)$, from the asymptotic decay of large Wilson loops $W^{a b, 2}(R, T)$ :

$$
W^{a b, 2}(R, T) \propto \exp \left(-V^{a b, a d j}(R) T\right)+\cdots \quad .
$$


We expect the string tension extracted from $V^{a b, a d j}(R)$ to approximate the adjoint string tension which can be defined at intermediate distances. This expectation is supported by an investigation of the abelian projection for the adjoint representation in the limit of large $N_{c}$ [20, 21].

\section{$3 \quad$ Efficient gauge fixing}

\subsection{Overrelaxed simulated annealing algorithm}

As has been pointed out above the abelian projection procedure requires (partial) gauge fixing. The differential gauge condition Eq. (3), supplemented by the constraint of FadeevPopov operator positivity, is equivalent to finding a maximum of the lattice functional, Eq. (1). Besides absolute maxima — which can in principle be degenerate even beyond trivial gauge transformations, such as constant ones and transformations within the maximally abelian subgroup - the functional $F(U)$ can have any number of local maxima. This feature resembles the Gribov problem of continuum gauge theories [11. While degenerate absolute maxima, at least for Landau gauge, may be safely ignored [22], imperfect gauging (where the system is stuck on some local maximum) may lead to fake physics results 23]. The aim of any reliable analysis must therefore be to drive the system by appropriate gauge procedures into local maxima as close as possible to the absolute ones. This would help to reduce systematic uncertainties due to gauge fixing ambiguities. A similar approach of gauge fixing in numerical simulations, motivated by global gauge fixing conditions [24, 25], has been advocated for Landau and Coulomb gauges in Ref. [26].

Traditionally, the relaxation plus overrelaxation (RO) algorithm has been employed for MA gauge fixing. In Ref. [27], we reported on the implementation of simulated annealing (SA), a technique that has been proven to be very useful in handling various optimization problems. In this SA algorithm [28, 29] the functional $F(U)$ is regarded as a "spin action",

$$
S(s)=F\left(U^{g}\right)=\frac{1}{8 V} \sum_{n, \mu} \operatorname{Tr}\left(s_{n} U_{n, \mu} s_{n+\mu} U_{n, \mu}^{\dagger}\right)
$$

where $s_{n}=g_{n}^{\dagger} \sigma_{3} g_{n}$ resemble spin variables. The lattice fields $U_{n, \mu}$ play the rôle of (almost) random local couplings. Maximizing the functional $F\left(U^{g}\right)$ is equivalent to decreasing the auxiliary temperature $T$ of the statistical system with partition function

$$
Z=\sum_{\left\{s_{n}\right\}} \exp \left(\frac{1}{T} S(s)\right) \quad .
$$

One starts with equilibrating this spin glass at high temperature. Subsequently, $T$ is decreased adiabatically. It is evident that in the limit $T \rightarrow 0$ the system approaches its 
ground state, i.e. the maximal value of $S$. The merits of SA can be phrased in the language of solid state physics: standard relaxation that corresponds to fast cooling might cause defects which are avoided in the adiabatic cooling procedure of SA. In order to enhance the adiabatic movement of the spin variables through phase space we complement simulated annealing with overrelaxation (OSA).

Our procedure consists of three steps:

1. Thermalization at $T=2.5$.

2. Gradual decreasing of $T$ down to $T=0.01$.

3. Final maximization by means of the RO algorithm.

In steps 1 and 2 an overrelaxation transformation is performed at six consecutive lattice sites and heatbath is applied to the seventh. Within step 2, every time when the heatbath update is applied to a site, the temperature is lowered by a quantum $\delta T$. For our large volume studies $\left(V=32^{4}\right)$, a variant of this algorithm (suitable for parallel computers like the Connection Machine CM-5 where sites are visited in lexicographical ordering within subcubes of $2^{4}$ sites each) has been employed. The combined effect of local overrelaxation plus local temperature reduction is to cut the number of cooling sweeps while remaining close to equilibrium.

For the initial thermalization at $T=2.5,20$ sweeps have been performed. Within the temperature range $2.5 \geq T \geq 0.1, \delta T(T)$ has been tuned such that the spin action increased about linearly with the number of iteration sweeps. This has been realized by subdividing this range into 24 intervals of width $\Delta T=0.1$.

The corresponding differences of the action $\Delta S(T)=S(T)-S(T-\Delta T)$ have been computed on equilibrated configurations and were found to be very stable against statistical fluctuations among different Monte Carlo (MC) configurations. We found practically no volume dependence and only a moderate impact from variations of the gauge coupling, $\beta$. The number of sweeps (out of a fixed total number) to be performed within each interval $(T-\Delta T, T]$ was chosen to be proportional to $\Delta S(T)$ and, subsequently, the corresponding value of $\delta T(T)$ has been determined. Within the region $0.1>T \geq 0.01$, 50 additional sweeps have been performed. Finally, the RO algorithm has been applied till a convergence criterion was satisfied?.

\footnotetext{
${ }^{2}$ The iterations have been stopped as soon as all rotations of $s_{n}$ among the lattice sites within a maximization sweep were equal to identity within single precision numerical accuracy.
} 


\subsection{Tuning the gauge fixing algorithm}

Prior to the large volume production runs, we compared the RO gauge fixing algorithm with three different OSA variants, using a $12^{4}$ lattice at $\beta=2.43$. The OSA variants employ three different cooling schedules at step 2 of our procedure: 250 (OSA1), 500 (OSA2) and 1000 (OSA3) total sweeps. The standard procedure (RO) has been applied with identical convergence criterion. We collected 30 statistically independent equilibrated $\mathrm{MC}$ configurations and produced 10 random gauge copies from each of them as inputs for the four algorithms.

Consider a gauge dependent abelian quantity $A$. In the following, we denote $\bar{A}$ to be the average over gauge copies and $\langle A\rangle$ to be the statistical average. In Table 1, we compare the output of the four algorithms for various quantities. $\delta_{s d}^{2}=\left\langle\overline{F^{2}}-\bar{F}^{2}\right\rangle$ denotes the scatter of the maximized value of the functional among gauge copies. An ideal algorithm would always yield $F=F_{\max }$, i.e. $\delta_{s d}=0 . \rho_{\operatorname{mon}}=1 /(4 V)\left\langle\sum_{n, \mu} k_{n, \mu}\right\rangle$ denotes the so-called monopole density. $W_{i j}$ are abelian $i \times j$ Wilson loops and $K^{a b}$ is the abelian string tension in lattice units.

The comparison of $\langle\bar{F}\rangle$ and $\delta_{s d}$ reveals that anyone of our OSA schedules is superior to the standard $\mathrm{RO}$ algorithm, with the longest schedule yielding the best results. The OSA2 and OSA3 algorithms perform equally on the level of statistical errors. In terms of total computer time spent with a scalar code, OSA2 is about a factor two slower than RO. OSA1, still being an improvement, is only $30 \%$ slower than RO. In our parallel implementation on a Connection Machine CM-5, OSA1 was found to run even slightly faster than $\mathrm{RO}$.

The lesson from Table 1 is that physical results can be substantially corrupted by inadequate gauge fixing. The functional $F$ turns out to be correlated with Wilson loops and anti-correlated with the monopole number and abelian string tension.

By applying state-of-the-art smearing techniques on the spatial transporters of abelian Wilson loops, we have been able to compute the abelian ground state potential. In Fig. 1 these potentials are displayed for the RO (used by all previous authors for MA gauge fixing) and the OSA3 algorithms with only one gauge copy used in both cases. In addition, the copy with largest functional among all OSA3 copies has been chosen on each configuration as our best estimate of the "true" maximum (circles). From this potential we obtain $K_{\text {best }}^{a b}=.0478(38)$ as an estimate of the abelian string tension[?. From Fig. 11 it is evident that this value will be overestimated by about $30 \%$ by use of the standard procedure (see also Table (1). Even our most expensive algorithm OSA3 yields a value that is off by about one statistical standard deviation from our best estimate $\left(K_{\mathrm{OSA} 3}^{a b}=.0536(30)\right)$.

\footnotetext{
${ }^{3}$ Within this section, all errors have been obtained by the jackknife procedure.
} 
In order to study the scaling properties, the investigations have been partly repeated on a lattice with nearly equal physical size but smaller lattice resolution $\left(16^{4}\right.$ at $\left.\beta=2.5115\right)$. The OSA2 and RO algorithms have been applied to 10 copies on 20 configurations, each. The qualitative properties are seen to be the same. Again, the string tension obtained after application of the $\mathrm{RO}$ algorithm is drastically overestimated: $K_{\mathrm{RO}}^{a b}=.0362(15)$ versus $K_{\text {best }}^{a b}=.0305(19)$. Since the non-abelian string tension at this $\beta$ value turned out to be $K=.0325(12)$, this difference is relevant to the physical conclusions drawn.

We conclude that the quality of MA gauge fixing (in terms of the value of $F\left(U^{g}\right)$ and the scatter of results among different gauge copies) can be significantly improved by applying the OSA algorithm, without any cost in computer time. Our systematic study of non gauge invariant quantities revealed that such improvement is in fact mandatory: for a reliable extraction of the abelian potential and other observables one must carefully eliminate biases from incomplete gauge fixing. An extension of the method to other gauges (e.g. Landau gauge) is straight forward.

\section{Biases from Gribov copies}

\subsection{Simulation technicalities}

Our main simulations have been performed on $32^{4}$ lattices at $\beta=2.5115$. As a first step, test runs with different OSA cooling schedules (as explained in the previous section) have been performed on four gauge copies, generated from two thermalized configurations with the above simulation parameters. We have chosen the following schedules for the temperature degrading sweeps: $N_{s}=250+50, N_{s}=500+50, N_{s}=1000+100, N_{s}=$ $2000+100$, and $N_{s}=5000+200$. The first numbers denote the sweeps spent within the linear region $(0.1<T<2.5)$. The latter numbers correspond to the sweeps applied within the interval $0.01<T<0.1$. Finally, direct maximization sweeps $(T=0)$ have been applied. Depending on the configuration and cooling schedule typically 10-100 such steps had to be performed until the rest vector criterion was satisfied.

The hysteresis curves of the spin action, $F$, as a function of the temperature, $T$, are displayed in Fig. 2. The upmost curve corresponds to the longest schedule. The scatter within each curve (which is not visible on the scale of the figure) is due to the four gauge copies generated and indicative for the (small) statistical uncertainty. The differences between the curves indicate that even for $N_{s}>2000$ thermal equilibrium is not yet reached.

For our final run, we decided to apply 1100 gauge fixing sweeps as a compromise between effort and outcome. This choice allows to generate a few local maxima of $F(U)$ on each configuration and subsequently to select the best out of them. As will be described 
below, the availability of several local maxima enables us to estimate systematic biases due to incomplete gauge fixing.

\subsection{Error estimates}

In order to estimate systematic errors induced by an incomplete gauge fixing procedure, we have generated $N=20$ random gauge copies on each of $N_{c}=30$ gauge configurations. Subsequently, these copies have been fixed to the MA gauge. Each abelian configuration (i.e. each gauge copy) $C_{j}(i)=\left\{\theta_{\mu}^{(j, i)}(n): \mu=1, \ldots, 4, n \in V\right\}$ is labeled in the following way: $j$ runs from one to the number of gauge copies, $N$, while $i$ runs from 1 to the number of Monte Carlo generated $S U(2)$ gauge configurations, $N_{c}$. On each of these copies, abelian quantities $A\left(C_{j}(i)\right)$ are measured, where $A$ denotes either a (smeared) Wilson loop, the plaquette or the monopole number. In addition, the values of the gauge fixing functional $F\left(C_{j}(i)\right)$ are stored. All gauge copies (on a given configuration) are sorted by the value of this functional:

$$
F\left(C_{1}\right) \leq F\left(C_{2}\right) \leq \cdots \leq F\left(C_{N}\right)
$$

We are now prepared to investigate, which value of $A$ we would have obtained on the "best" out of $m \leq N$ copies. To answer this question one has to select $m$ random copies out of the $N$ copies that have been generated in total and subsequently extract $A$ from the copy with largest functional $F$, i.e. largest index $i$. Averaging over all possible choices yields

$$
A_{m}=\left(\begin{array}{c}
N \\
m
\end{array}\right)^{-1} \times \sum_{j=m}^{N}\left(\begin{array}{c}
j-1 \\
j-m
\end{array}\right) A\left(C_{j}\right)
$$

as the "average best copy" expectation of $A$ on subsets of size $m$ where the gauge configuration index has been omitted. As expected, the above formula corresponds to the average over all copies for the special case $m=1$ while for $m=N$ one obtains the value of $A$ on the "best" copy. Quantities like the abelian potential can be computed from averages of these $A_{m}$ 's over the $N_{c}$ configurations. The situation is visualized in Fig. 3 for the gauge functional $F$ itself. Throughout the rest of this paper, all statistical errors have been computed by the bootstrap procedure.

We wish to determine the expected deviation of the estimate $\left\langle A_{m}\right\rangle=\frac{1}{N_{c}} \sum_{i=1}^{N_{c}} A_{m}(i)$ from the true value $\left\langle A_{\infty}\right\rangle$, related to an absolute maximum. To estimate this bias we make the following assumptions:

- The absolute maximumf is unique.

\footnotetext{
${ }^{4}$ The term "absolute maximum" is to be understood modulo trivial degeneracies, due to constant
} 
- The algorithm is in principle able to reach the absolute maximum $F_{\text {global }}$, i.e. $F_{\infty}=$ $F_{\text {global }}$.

- In the neighborhood of the absolute maximum, $\left\langle A_{m}\right\rangle$ approaches $\left\langle A_{\infty}\right\rangle$ as a monotonous function of $\left\langle F_{\infty}-F_{m}\right\rangle$.

The first assumption is supported by Zwanziger's proof of the non-degeneracy of absolute maxima within the interior of the fundamental modular region in Landau gauge on the lattice [22] while the third assumption is supported by numerical evidence as all our observables exhibit strong correlations with the average value of the functional.

The difference, $\Delta_{m}^{A}=\left\langle A_{\infty}-A_{m}\right\rangle$, is to be seen as the very bias on $A_{m}$ from incomplete gauge fixing. The statistical uncertainty on this bias, on the other hand, is nothing else but the systematic error on our final result. As to its $m$-dependence, we start from the ansatz

$$
\Delta_{m}^{A}=c_{1} \exp \left(-d_{1} m\right)+\cdots
$$

for the (large $m$ ) asymptotic behavior, to be tested against the data. Accordingly, we can fit our data to the form

$$
\left\langle A_{m}\right\rangle=\left\langle A_{\infty}\right\rangle-c_{1} \exp \left(-d_{1} m\right)+\cdots
$$

where $\left\langle A_{\infty}\right\rangle, c_{1}$ and $d_{1}$ are free parameters. The statistical errors on $\left\langle A_{m}\right\rangle$ imply a large uncertainty on $\left\langle A_{\infty}\right\rangle$. However, due to strong correlations among the data, the differences $\Delta_{m}^{A}$ can be obtained quite accurately. In view of our limited statistics for the study of gauge fixing ambiguities $\left(N_{c}=30\right)$ we have not applied full correlated fits. Nonetheless, some of the correlations have been taken into account by fitting the differences

$$
\Delta^{A}(N, m)=\left\langle A_{N}-A_{m}\right\rangle=\Delta_{N}^{A}\left(e^{(N-m) d_{1}}-1\right) \quad, \quad \Delta_{N}^{A}=c_{1} e^{-N d_{1}}
$$

rather than following Eq. 40. The result of such a fit (from $m=5$ onwards) is visualized in Fig. 4 . On the basis of this fit the bias on $A_{m}$ can be traced back into the region of small $m$, in the form $\Delta_{m}^{A}=\Delta_{N}^{A}+\Delta^{A}(N, m)$.

Results from the above procedure for the functional, $F$, the abelian plaquette action, $S=1-\left\langle W^{a b}(1,1)\right\rangle$, and the monopole density $\rho_{\text {mon }}$ for $m=1,10,20$ as well as for the extrapolated value $(m=\infty)$ are compiled in Table 2. We find that the proposed error analysis is a powerful tool to obtain reliable estimates for biases. For instance, when

gauge transformations, gauge transformations within the unfixed diagonal $U(1)$ subgroup and the $2^{4}$ degenerate maxima from $Z_{2}$ center group transformations within hyperplanes perpendicular to the four possible lattice orientations, which only affect expectation values of Polyakov line-like operators but leave the spectrum invariant. 
selecting the best out of 10 copies generated by our OSA algorithm, we find $\Delta F_{10}=$ $0.000055(10), \Delta S_{10}=-0.000037(30)$, and $\Delta \rho_{\text {mon, } 10}=-0.000015(15)$.

We would like to emphasize that, by computing the biases, we have found a way to extrapolate values, obtained on local maxima, to an absolute maximum. The accuracy of all computations is limited by the statistical error on the biases.

\subsection{Application to the abelian potential}

The abelian potential, $V_{m}^{a b}(\mathbf{R})$, as well as the abelian string tension, $K_{m}^{a b}$, have been computed for various $m \leq N$, by use of the method described in Section 5.1. We find the abelian string tension, $K_{m}^{a b}$ to be anticorrelated with the gauge fixing functional $\left(K_{m}^{a b}<K_{m-1}^{a b}\right)$. The potential values themselves exhibit a systematic, but statistically insignificant, drift.

The correlation between the fitted abelian string tension and $m$ can be read off

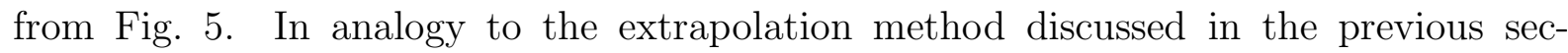
tion, $\Delta^{K}(N, m)$ is fitted to an exponential ansatz. As a result, the systematic bias, $\Delta_{m}^{K}=\Delta_{N}^{K}-\Delta^{K}(N, m)$ is obtained as a function of $m$ (Fig. [1). The statistical errors on $N_{c}=30$ configurations (solid line) are indicated in the figure, as well as the error on the final statistical ensemble of 108 configurations, obtained on the best out of 10 gauge copies (horizontal dashed line).

For the final production runs we decided to choose $m=10$ as a reasonable compromise. For this $m$-value the expected statistical error on the string tension matches both, the size and the uncertainty of its bias.

Contrary to the case of the string tension (Fig. 6), we find no statistically significant bias on the values of the Coulomb coefficient $e$ and the self energy $V_{0}$ which are dominated by the short range part of the potential.

Results on the abelian string tension from different numbers of gauge copies are collected in Table 2 (last row). The bias on 10 gauge copies is $\Delta_{10}^{K}=-0.00078(72)$ while the expected bias on one gauge copy would have been $\Delta_{1}^{K}=-0.0021(9)$.

\section{Physics results}

Our main measurements have been performed on the same lattice volume and $\beta$ value as the investigation of systematic gauge fixing errors, presented in the previous section $\left(V=32^{4}, \beta=2.5115\right)$. This enables us (a) to correct the results for the estimated

\footnotetext{
${ }^{5}$ The statistical errors come out to decrease with decreasing $m$ due to the procedure of combinatoric averaging, Eq. 38 .
} 
biases and (b) to include systematic uncertainties into the final errors. The spatial lattice extent corresponds to $2.7 \mathrm{fm}$ in physical units where the scale has been obtained from the value $\kappa=K a^{-2}=(440 \mathrm{MeV})^{2}$ for the string tension. This ensures finite size effects on the potentials to be negligible. The $\beta$ value was chosen sufficiently large to be within the scaling region, and in respect to future finite temperature studies (The critical temperature comes out to be $T_{c}=(8 a)^{-1}$ [30]).

The hybrid-overrelaxed algorithm [31] with Fabricius-Haan heatbath sweeps [32] has been applied to update the gauge fields. Subsequent configurations are separated by 200 such sweeps and have been found to be statistically independent. Abelian projection and measurements have been performed on 108 such gauge configurations. The following abelian observables (i.e. quantities expressed in terms of abelian gauge fields, $\theta_{n, \mu}$ ) have been investigated: charge one and two potentials and string tensions, photon and monopole contributions to the abelian string tension and the abelian monopole density.

\subsection{Abelian and non-abelian static potentials}

We have computed the abelian potential on 108 configurations as well as the non-abelian potential on 644 configurations by use of one and the same analysis method to allow for direct comparison of results.

Our final results on the abelian potential have been obtained on the best out of 10 gauge copies. The systematic biases are estimated from 30 configurations with 20 gauge copies on each. Both, abelian and non-abelian potentials have been obtained from Wilson loops with smeared spatial paths in order to enhance the overlap of the $Q \bar{Q}$ creation

operator with the $Q \bar{Q}$ ground state [19]. To reduce statistical fluctuations, we have analytically integrated out temporal links of the non-abelian Wilson loops [33.

We found good results by iteratively applying the smearing procedure,

$$
\theta_{n, j} \rightarrow \arg \left[\alpha \exp \left(i \theta_{n, j}\right)+\sum_{k \neq j} \exp \left(i\left(\theta_{n, k}+\theta_{n+\hat{k}, j}-\theta_{n+\hat{j}, k}\right)\right)\right],
$$

to spatial link angles, i.e. by substituting the corresponding $U(1)$ element by a linear combination of the previous one and the sum of the four spatial staples, enclosing it. We have chosen the parameter value $\alpha=1$ and 150 iterations. Rectangular Wilson loops, constructed from such smeared spatial links, can be decomposed into a linear combination of various loops with fixed corners but different spatial connections. For extraction of the non-abelian potential, we applied a similar procedure on the $S U(2)$ link variables with the parameter value $\alpha=2$. 
For large temporal extent, $T$, the potential can be extracted from the asymptotic expectation,

$$
\langle W(\mathbf{R}, T)\rangle=C(\mathbf{R}) \exp (-V(\mathbf{R}) T)+\cdots,
$$

where $C(\mathbf{R})$ denote the ground state overlaps. For finite values of $T$, we define the following approximants to overlaps and potentials, which will monotonously decrease towards their asymptotic values,

$$
V_{T}(\mathbf{R})=\log \left(\frac{\langle W(\mathbf{R}, T)\rangle}{\langle W(\mathbf{R}, T+1)\rangle}\right) \quad, \quad C_{T}(\mathbf{R})=\langle W(\mathbf{R}, T)\rangle \exp \left(V_{T}(\mathbf{R}) T\right) \quad .
$$

By comparing results on the potential approximants (abelian and non-abelian) between the on-axis direction with those, obtained on five different off-axis directions, we find $S O(3)$ rotational invariance to be restored (within statistical accuracy) for $R \geq 3$. In both cases, the data for $R \geq 2 \sqrt{3}$ are well described (for $T \geq 3$ ) by the parametrization,

$$
V(R)=V_{0}+K R-\frac{e}{R}
$$

By fitting different $T$-approximants to the potential with this parametrization, we obtain approximants to the string tension, $K_{T}$. By demanding plateaus of $C_{T}(R), V_{T}(R)$ and $K_{T}$ for $T \geq T_{\min }$, we find $T_{\min }=4$ and $T_{\min }=6$ for the abelian and non-abelian potentials, respectively. The different onset of asymptotics is due to superior overlaps with the ground state in case of the smeared abelian Wilson loops. The self energy term, $V_{0}^{a b}$ is much smaller than its abelian counterpart $V_{0}$, resulting in larger numerical values of the corresponding abelian Wilson loops. These two effects are among the reasons for reduced statistical errors on the abelian potential and fit parameters. We take the $T_{\min }$ approximants to potential values and fit parameters as our asymptotic results. To avoid systematic effects from the fit range creeping into the comparative interpretation of results, we select a universal $R$-range for all fits, $2 \sqrt{3} \leq R \leq 16$. This provides us with 45 onand off-axis data points.

Our results on the fit parameters are in qualitative agreement with previous publications [8]. In particular, the self energy $V_{0}^{a b}$ and Coulomb coefficient $e^{a b}$ come out to be by more than a factor two smaller than their non-abelian counterparts while the abelian string tension is found to be close to the non-abelian one (see Table 3 and Fig. 7). As pointed out above, all systematic uncertainties are understood and under control in the present investigation, in particular the approach to the $T \rightarrow \infty$ limit. The biases due to gauge fixing ambiguities have been neglected in previous studies but turn out to be important as demonstrated in Sections 3 and 4.

The value of the abelian string tension comes out to be $K^{a b}=0.0305(3)$ on the best out of 10 OSA gauge copies, which nicely agrees with the value $K^{a b}=0.0305(19)$, as obtained 
on a $16^{4}$ lattice (Section 3) at the same $\beta$ value. By including bias and uncertainty of the bias, we end up with $K^{a b}=0.0297(8)$, where the error includes the systematic uncertainty. This amounts to the ratio $K^{a b} / K=0.92(4)$ (see Table B).

Given the precision of our data and analysis tools, we find a significant deviation between the string tension of $S U(2)$ gauge theory and its MA content at $\beta=2.5115$. Further measurements at different lattice spacings are required to decide whether this ratio approaches unity in the continuum limit, as expected from abelian dominance. However, for the time being, at finite lattice spacing, the string tension from the abelian projected theory appears to be definitely smaller than its non-abelian counterpart.

\subsection{Decomposition of the abelian potential}

We have tried two different approaches to disentangle monopole and photon contributions to the potential.

The first method rests on the determination of the monopole part from elementary monopole currents, $k_{n, \mu}$. The potential estimators $V_{T}^{\text {mon }}(R)$, extracted from $\left\langle W_{\text {mon }}(R, T)\right\rangle$ (see Eq. (18)) at fixed $R$ have been observed to increase with $T$, which means that the coefficients $C^{\prime}(R)$ of the decomposition,

$$
\left\langle W^{m o n}(R, T)\right\rangle=C(R) \exp \left(-V^{m o n}(R) T\right)+C^{\prime}(R) \exp \left(-V^{\prime m o n}(R) T\right)+\cdots \quad,
$$

are not necessarily positive. This unpleasant feature, in conjunction with the requirement of $T \gg R$ makes this procedure of extracting $V^{m o n}(R)$ unreliable.

As a way out, we started from an alternative representation of the factorization property to $W^{a b}$ (Eqs. (26) and (27)), which allows decoupling of excited states through smearing of spatial links $\theta_{n, j}^{\text {mon }}$. In this second approach, the abelian configuration has been fixed for technical reasons to Landau gauge,

$$
\partial_{\mu} \sin \left(\theta_{n, \mu}\right)=0
$$

prior to evaluation of $\theta_{n, \mu}^{\text {mon }}$ (Eq. (26)). This reduces the number of plaquettes with nonzero $m_{n, \mu \nu}$ and, thus, computer time by more than one order of magnitude. It should be noted that - though $\theta_{n, \mu}^{\text {mon }}$ transforms under $U(1)$ gauge transformations Eq. (5) - $W^{\text {mon,fv as }}$ well as $W^{p h}$ are gauge invariant quantities.

After evaluation of $\theta_{n, \mu}^{\text {mon }}$ and $\theta_{n, \mu}^{\prime}=\theta_{n, \mu}-\theta_{n, \mu}^{\text {mon }}$, smearing has been applied and the corresponding potentials have been extracted. For small $R$, the results as obtained from the two methods are found to agree, while at large $R$, plateaus in $T$ could only be established for potential estimates extracted from smeared monopole and photon Wilson loops. 
As pointed out in Section 2, the full factorization ansatz may include finite volume contributions. However, no statistically significant such effects have been found by comparison of unsmeared $W^{m o n, f v}$ with $W^{m o n}$ (calculated by use of elementary monopole currents $k_{n, \mu}$ via Eq. (18) for all realized $R$ and $T$ values, on a configuration by configuration basis). Thus, we conclude that finite size effects can indeed be neglected on our lattice volume.

The resulting potentials as well as $V^{a b}$ are displayed in Fig. 8 and the parameter values as obtained from fits according to Eq. (45) are quoted in Table 4 . The corresponding values for the abelian potential are included as well, where we have omitted the bias from gauge fixing ambiguities, to allow for a direct comparison.

One can see from Fig. 8 that the photon part $V^{p h}$ does not contribute to the string tension. Therefore, in MA projection only the monopole part of the abelian gauge fields, $\theta_{n, \mu}^{m o n}$, gives rise to the flux tube. A comparison of $V^{a b}$ with $V^{m o n, f v}+V^{p h}$ reveals a qualitative agreement, implying the approximate validity of the factorization ansatz. The string tension of the monopole contribution amounts to $(95 \pm 1) \%$ of the full abelian string tension. The approximate decomposition of the abelian static potential into monopole and photon parts gives evidence for the interaction term between monopoles and photons within the corresponding effective action to be weak.

We have also attempted to fit the photon part of the potential to the ansatz,

$$
V^{p h}(\mathbf{R})=V_{\text {self }}-f G_{L=32}(\mathbf{R}) \quad, \quad G_{L}(\mathbf{R})=\frac{4 \pi}{V} \sum_{\mathbf{k} \neq \mathbf{0}} e^{i \mathbf{k R}} D(\mathbf{k}, 0)
$$

for various fit ranges. $G_{L}(\mathbf{R})$ is the Coulomb potential on the lattice which approaches $1 / R$ in the infinite volume limit $(L \rightarrow \infty)$ for large (lattice) $R$. Lattice artefacts turned out to be well parameterized by this functional form. As a result we quote $V_{\text {self }}=0.2513(3)$ and $f=0.130(30)$, obtained on the fit range $3 \leq R \leq 12.12$. Data and fit curve are visualized in Fig. 9. The fit range turned out to be correlated with the parameter value $f$ in so far as $f$ tended to be larger if large $R$ values had been included and smaller for small $R$ values. This is also evident from the figure and might be interpreted as a relict of asymptotic freedom within the abelian projected gauge theory. From tree level perturbation theory, one might expect $f=V_{\text {self }} / G_{L=32}(\mathbf{0}) \approx 0.0814$, which is smaller than the fitted $f$ quoted above, in accordance with a running coupling interpretation.

The monopole contribution to the abelian potential $V_{l=2}^{a b}$ has been extracted from extended monopoles of size $l=2$ as well, by use of the method, introduced in Section 2 (Eq. (24)). By computing the potential from the corresponding Wilson loops (which has been done in Ref. [16] the first time), we found the approximants to decrease monotonously in $T$. The value of the monopole string tension turned out to be slightly smaller than the one extracted from elementary monopoles $\left(K_{l=2}^{\text {mon }}=0.0271(3)\right.$ instead of $K^{\text {mon }}=$ 
0.0290(3), c.f. Table 4), which might indicate that a small portion of the string tension is due to monopole structures of smaller extent.

The new method of computing the monopole contribution to the potential by combining Eqs. (26) and (27) with the smearing method has the advantage of large ground state overlaps. Also, the potential can easily be computed for off-axis points while the blocking method even reduces the number of on-axis points at which the potential can be measured by a factor two. Notice, that $V^{\text {mon,fs }}$ obtained by use of the smearing method is identical to $V^{m o n}+V^{f s}$, as computed in the traditional way from elementary monopole currents, while monopole structures on the scale of a lattice spacing are neglected in the blocking method.

\subsection{Charge two case}

In order to extract the charge two potential $V^{a b, 2}(R)$ (Section 2.4), smeared charge two Wilson loops, $W^{a b, 2}(R, T)$ have been evaluated. The data for the fundamental and charge two abelian potentials are displayed in Fig. 10 (with the fitted self energies being subtracted).

Flux tube models lead to the expectation $K^{\text {adj }} / K=8 / 3$ for the ratio of the adjoint over the the fundamental string tension?. This value was qualitatively supported by numerical data [34, 35]. However, recent results [36, 37] indicate that at intermediate to large distances the above ratio tends to be somewhat smaller than $8 / 3$. We suggest the slope of the charge two potential, $K^{a b, 2}$ to constitute the abelian projection counterpart to the adjoint string tension and thus expect the ratio $K^{a b, 2} / K^{a b}$ to agree with results on $K^{a d j} / K$. However, the lack of high precision data on the latter ratio prevents us from a quantitative test of this assumption. From our data, we find the value $K^{a b, 2} / K^{a b}=2.23(5)$ significantly smaller than $8 / 3$.

For small $R$, perturbation theory yields the same $8 / 3$ ratio between the adjoint and the fundamental potential, which differs from the expected ratio between abelian charge two and charge one potentials, where one naively would expect a factor $2^{2}=4$. We indeed obtain $V_{0}^{a b, 2} / V_{0}^{a b}=3.96(7)$ and $e^{a b, 2} / e^{a b}=4.0(5)$ for the fit parameters that are sensitive to short range physics. We have included the curve $8 / 3 K^{a b}-4 e^{a b} / R$ into Fig. 10, which corresponds to $S U(2)$ Casimir scaling for the linear part of the potential and to the perturbative $U(1)$ expectation for its Coulomb part.

\footnotetext{
${ }^{6}$ Of course, the adjoint string tension is only an effective quantity since, at large distance, string breaking is expected to set, caused by screening from the creation of glueball pairs.
} 


\subsection{Monopole density}

Monopole densities for elementary as well as for extended monopole currents of size $l=2$ have been evaluated. The density of monopoles of size $l$ in physical units is defined as [

$$
\rho_{\operatorname{mon}}^{(l), p h}=\frac{1}{4 V \cdot(l a)^{3}} \sum_{n, \mu}\left\langle\left|k_{n, \mu}^{(l)}\right|\right\rangle
$$

We obtain the following results for the monopole densities, converted into units of the string tension as measured in the present investigation:

$$
\frac{\rho_{\mathrm{mon}}^{p h}}{K^{3 / 2}}=1.962(4) \quad, \quad \frac{\rho_{\mathrm{mon}}^{(2), p h}}{K^{3 / 2}}=1.269(2) .
$$

The value for elementary monopole currents has been corrected by its systematic bias from incomplete gauge fixing.

In order to relate our results to those obtained in previous publications, we compare our value on the density of elementary monopoles with the value $\rho_{\text {mon }}^{p h} / K^{3 / 2}=2.11(2)$ from Ref. [38] for a $16^{4}$ lattice at $\beta=2.5$, which is close to our coupling constant, $\beta=2.5115$. Note, that we have rescaled the result of Ref. [38] into units of the string tension as obtained in Ref. [19]. The result of Ref. [38] turns out to be consistent within errors with results from other authors [39, 17, 40] while our value is significantly smaller. Since finite size effects are negligible for the lattice extents under consideration, the difference seems to be due to our improved OSA gauge fixing algorithm. A similar discrepancy is observed for extended monopoles. In this case our value, Eq. (50), should be compared

with $\rho_{\text {mon }}^{(2), p h} / K^{3 / 2}=1.32$, as obtained on a $24^{4}$ lattice at $\beta=2.5$ by means of the standard OR gauge fixing procedure [41].

\section{Summary and conclusions}

Let us summarize our main results and conclude:

- The present study is based on a self contained and self consistent analysis on the largest lattice volume that has been studied so far for this kind of simulations with comparatively high statistics. A systematic error analysis has been carried out for the first time in this context.

\footnotetext{
${ }^{7}$ The factor $l^{3}$ appears in the denominator of Eq. (49), due to averaging over the $l^{3}$ possible blocked sublattices.
} 
- To obtain reliable results in the abelian projected theory with MA gauge condition, one has to investigate and control the uncertainty that is inevitably introduced by the incomplete gauge fixing of numerical practice. The OSA algorithm has been shown to be a powerful tool for gauge fixing. A method for estimation of residual uncertainties is proposed.

- We have found $K^{a b}$ to be $(8 \pm 4) \%$ smaller than the non-abelian string tension at $\beta=2.5115$.

- Our investigation of the decomposition of the static abelian potential into monopole and photon parts confirms earlier observations at a higher confidence level. By applying a new method for extracting the monopole contribution to the potential, we have been able to extract the corresponding potentials and fit parameters reliably. The factorization has been found to work qualitatively, the monopole contribution accounting for the string tension within a margin of $5 \%$.

- We have calculated the abelian projection approximation for the adjoint string tension. Our result for ratio $K^{a b, 2} / K^{a b}$ is in qualitative agreement with numerical data on its non-abelian counterpart.

We believe that further computations at different $\beta$ values, provided all sources of errors are kept under control as in the present paper, will answer the question whether the abelian projected theory exactly reproduces the large distance behavior of the full theory in the continuum limit.

\section{Acknowledgements}

We thank the Deutsche Forschungsgemeinschaft for supporting the Wuppertal CM-5 project (grants Schi 257/1-4 and Schi 257/3-2) and the HLRZ for computing time on the CM-5 at GMD. GB, KS and MMP appreciate support by European Union contracts SC1* CT91-0642 and CHRX-CT92-0051. During completion of this work GB received funding by EU contract ERB-CHBG-CT94-0665. This work was supported in part by grant No. NJP000, financed by the International Science Foundation, by grant No. NJP300, financed by the International Science Foundation and by the Government of the Russian Federation. VB obtained partial support from DFG grant 436-RUS-113 and from Grant No. 93-02-03609, financed by the Russian Foundation for Fundamental Sciences. 


\section{Note added in proof}

After completion of the present paper, a preprint by G. Poulis 42 has been received, in which the author has demonstrated that one should expect $K^{a b, 2}$ and $K^{a d j}$ to coincide to the same extent as $K^{a b}$ and $K$ agree by use of reasonable approximations.

\section{References}

[1] G. 't Hooft, in 'High Energy Physics', Proceedings of the EPS International Conference, Palermo 1975, ed. A. Zichichi, Editrice Compositori, Bologna 1976.

[2] S. Mandelstam, Phys. Rep. C23 (1976) 245.

[3] A.M. Polyakov, Phys. Lett. 59B (1975) 82.

[4] T. Banks, R. Myerson, J. Kogut, Nucl. Phys. B129 (1977) 493.

[5] J. Smit, A.J. van der Sijs, Nucl. Phys. B355 (1991) 603.

[6] G. 't Hooft, Nucl. Phys. B190 [FS3] (1981) 455; G. 't Hooft, Phys. Scr. 25 (1982) 133.

[7] A.S. Kronfeld, G. Schierholz, U.-J. Wiese, Nucl. Phys. B293 (1987) 461; A.S. Kronfeld, M.L. Laursen, G. Schierholz, U.-J. Wiese, Phys. Lett. 198B (1987) 516.

[8] T. Suzuki, Nucl. Phys. B[Proc. Suppl.]30 (1993) 176.

[9] A. DiGiacomo, review talk presented at the International Symposium on Lattice Field Theory Lattice '95, Melbourne, 1995.

[10] T. Suzuki, I. Yotsuyanagi, Phys. Rev. D42 (1990) 4257.

[11] V.N. Gribov, Nucl. Phys. B139 (1978) 1.

[12] H. Min, T. Lee, P.Y. Pac, Phys. Rev. D32 (1985) 440.

[13] M.N. Chernodub, M.I. Polikarpov and A.I. Veselov, Phys. Lett. B342 (1995) 303.

[14] T.A. DeGrand and D. Toussaint, Phys. Rev. D22 (1980) 2478.

[15] J. Stack and R. Wensley, Nucl. Phys. B371 (1992) 597. 
[16] H. Shiba and T. Suzuki, Phys.Lett. B333 (1994) 461.

[17] J. Stack, S. Neiman and R. Wensley, Phys. Rev. D50 (1994) 3399.

[18] T.L. Ivanenko, A.V. Pochinsky and M.I. Polikarpov, Phys. Lett. B252 (1990) 631.

[19] G.S. Bali, C. Schlichter and K. Schilling, Phys. Rev. D51 (1995) 5165.

[20] L. DelDebbio, M. Faber and J. Greensite, Nucl. Phys. B414 (1994) 594.

[21] L. DelDebbio, M. Faber, J. Greensite and S. Olejnik, "Casimir Scaling vs. Abelian Dominance in QCD String Formation", preprint hep-lat/9510028.

[22] D. Zwanziger, Nucl. Phys. B412 (1994) 657.

[23] V. Bornyakov, V.K. Mitrjushkin M. Müller-Preussker and F. Pahl, Phys. Lett. B317 (1993) 596.

[24] C. Parrinello and G. Jona-Lasinio, Phys. Lett. B251 (1990) 175.

[25] D. Zwanziger, Nucl. Phys. B345 (1990) 461.

[26] S. Fachin and C. Parrinello, Nucl. Phys. B[Proc. Suppl.] 26(1992) 429.

[27] G. Bali, V. Bornyakov, M. Müller-Preussker and F. Pahl, Nucl. Phys. B[Proc. Suppl.] 42(1995) 852.

[28] S. Kirkpatrick, C.D. Gelatt Jr., M.P. Vecchi, Science 220 (1983) 671.

[29] V. Cerny, J. Opt. The. Appl. 45 (1985) 41.

[30] J. Fingberg, U.M. Heller, F. Karsch, Nucl. Phys. B302 (1993) 493.

[31] F.R. Brown and T.J. Woch, Phys. Rev. Lett. 28 (1987) 2394; R. Gupta, G.W. Kilcup, A. Patel, S.R. Sharpe, and P. de Forcrand, Mod. Phys. Lett. A3 (1988) 1367.

[32] K. Fabricius and O. Haan, Phys. Lett. B143, (1984) 459; A. Kennedy and B. Pendleton, Phys. Lett. B156 (1985) 393.

[33] G. Parisi, R. Petronzio and F. Rapuano, Phys. Lett. 128B (1983) 418.

[34] J. Ambjorn, P. Olesen and C. Peterson, Nucl. Phys. B240 (1984) 189.

[35] C. Michael, Nucl. Phys. B259 (1985) 58. 
[36] C. Michael, Nucl. Phys. B[Proc. Suppl.]26 (1992) 417.

[37] H. Trottier, Phys. Lett. B357 (1995) 193.

[38] V. Bornyakov, E.-M. Ilgenfritz, M.L. Laursen, V.K. Mitrjushkin, M. MüllerPreussker, A.J. van der Sijs, A.M. Zadorozhny, Phys. Lett. 261B (1991) 116.

[39] S. Hioki, S. Kitahara, S. Kiura, Y. Matsubara O. Miyamura, S. Ohno and T. Suzuki, Phys. Lett. B272 (1991) 326.

[40] L. Del Debbio, A. DiGiacomo, M. Maggiore and S. Olejnik, Phys. Lett. B267 (1991) 254.

[41] H. Shiba, private communication.

[42] G. Poulis, preprint hep-lat/9601013. 
Table 1: Comparison of gauge fixing algorithms.

\begin{tabular}{ccccc}
\hline & RO & OSA1 & OSA2 & OSA3 \\
\hline$\langle\bar{F}\rangle$ & $0.7370(2)$ & $0.7383(2)$ & $0.7387(2)$ & $0.7390(2)$ \\
$\delta_{\text {sd }}$ & $18 \cdot 10^{-5}$ & $13 \cdot 10^{-5}$ & $10 \cdot 10^{-5}$ & $8 \cdot 10^{-5}$ \\
$\rho_{\text {mon }}$ & $0.0218(2)$ & $0.0209(3)$ & $0.0207(3)$ & $0.0204(3)$ \\
$W_{11}$ & $0.7702(5)$ & $0.7716(5)$ & $0.7720(5)$ & $0.7723(5)$ \\
$W_{44}$ & $0.085(1)$ & $0.090(1)$ & $0.091(1)$ & $0.092(2)$ \\
$K^{a b}$ & $0.063(3)$ & $0.057(3)$ & $0.055(3)$ & $0.054(3)$ \\
\hline
\end{tabular}

Table 2: Dependence of results on the number of gauge copies, $m$, at $\beta=2.5115, V=32^{4}$, $N_{c}=30$.

\begin{tabular}{ccccc}
\hline & $m=1$ & $m=10$ & $m=20$ & $m=\infty$ \\
\hline$\langle F\rangle$ & $0.752641(34)$ & $0.752789(35)$ & $0.752822(36)$ & $0.752845(37)$ \\
$S$ & $0.202508(60)$ & $0.202299(64)$ & $0.202267(64)$ & $0.202261(66)$ \\
$\rho_{\text {mon }}$ & $0.011639(26)$ & $0.011528(30)$ & $0.011515(32)$ & $0.011513(33)$ \\
$K^{a b}$ & $0.0325(11)$ & $0.0311(13)$ & $0.0306(16)$ & $0.0303(17)$ \\
\hline
\end{tabular}

Table 3: Fit parameters for the static potentials in abelian projected $S U(2)$ (corrected by the estimated bias due to incomplete gauge fixing) and the potential of full $S U(2)$. Errors are systematic and statistical.

\begin{tabular}{ccc}
\hline & AP $S U(2)$ & $S U(2)$ \\
\hline$e$ & $0.095(11)$ & $0.252(24)$ \\
$V_{0}$ & $0.240(4)$ & $0.545(10)$ \\
$K$ & $0.0297(8)$ & $0.0325(12)$ \\
\hline
\end{tabular}

Table 4: Test of factorization of the abelian potential into monopole and photon parts. Biases from incomplete gauge fixing have been omitted throughout the table.

\begin{tabular}{cccccc}
\hline & $V^{a b}$ & $V^{\text {mon,fv }}+V^{p h}$ & $V^{\text {mon, fv }}$ & $V_{l=2}^{\text {mon }}$ & $V^{\text {ph }}$ \\
\hline$e$ & $0.095(6)$ & $0.068(5)$ & $-0.056(5)$ & $0.019(4)$ & $0.124(2)$ \\
$V_{0}$ & $0.240(3)$ & $0.232(2)$ & $-0.029(2)$ & $0.010(2)$ & $0.261(1)$ \\
$K$ & $0.0305(3)$ & $0.0291(3)$ & $0.0290(3)$ & $0.0271(3)$ & $0.00007(4)$ \\
\hline
\end{tabular}


Table 5: Static potential parameters for the abelian projections of the fundamental $(q=1)$ and adjoint $(q=2)$ representations of $S U(2)$.

\begin{tabular}{cccc}
\hline & $\mathrm{AP} S U(2)(q=1)$ & $\mathrm{AP} S U(2)(q=2)$ & $(q=2) /(q=1)$ \\
\hline$e$ & $0.095(6)$ & $0.376(36)$ & $3.98(48)$ \\
$V_{0}$ & $0.240(2)$ & $0.950(13)$ & $3.96(7)$ \\
$K$ & $0.0305(3)$ & $0.0682(11)$ & $2.23(5)$ \\
\hline
\end{tabular}

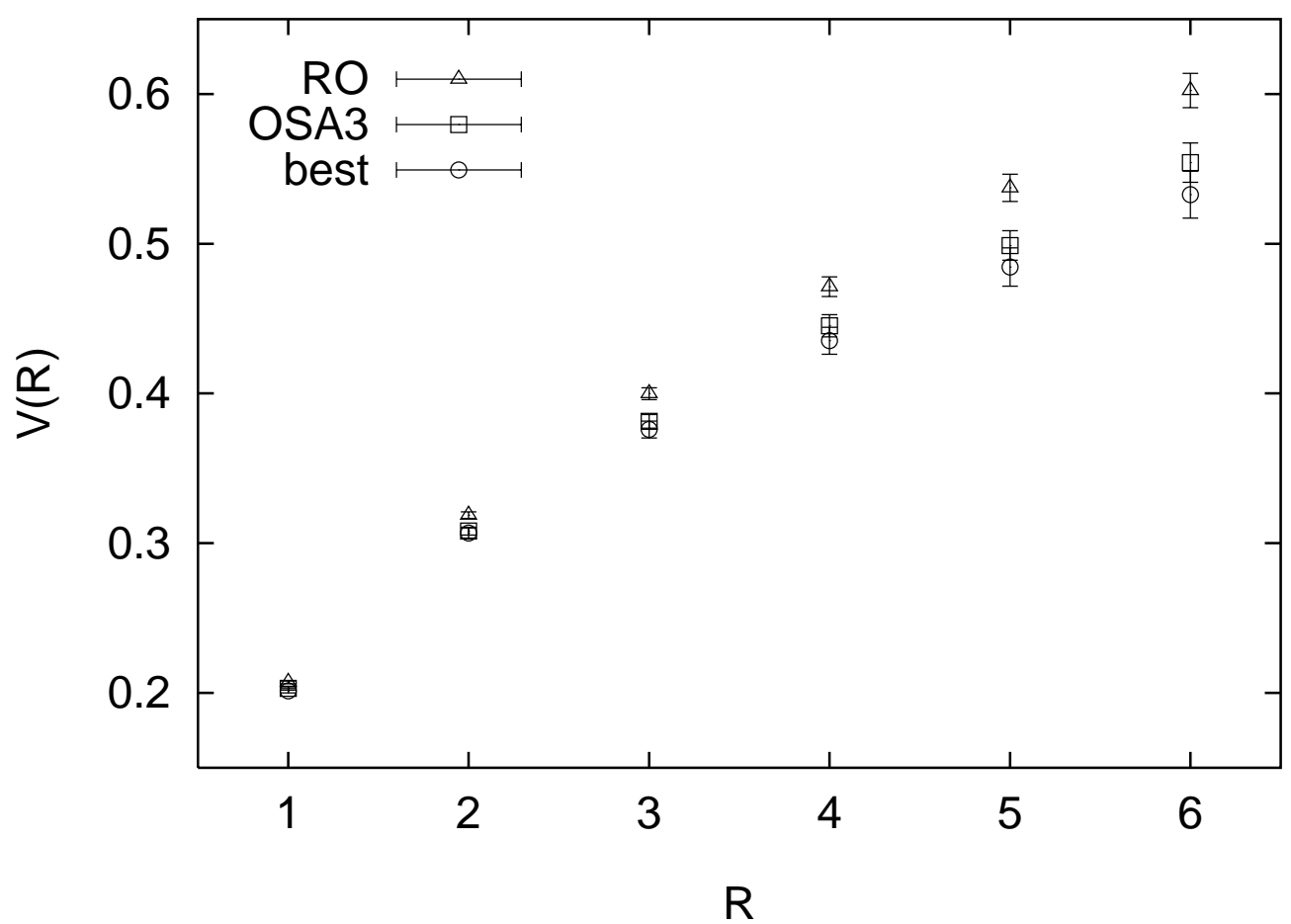

Figure 1: Abelian potentials from RO (triangles), OSA3 (squares) and "best" copy (circles). 


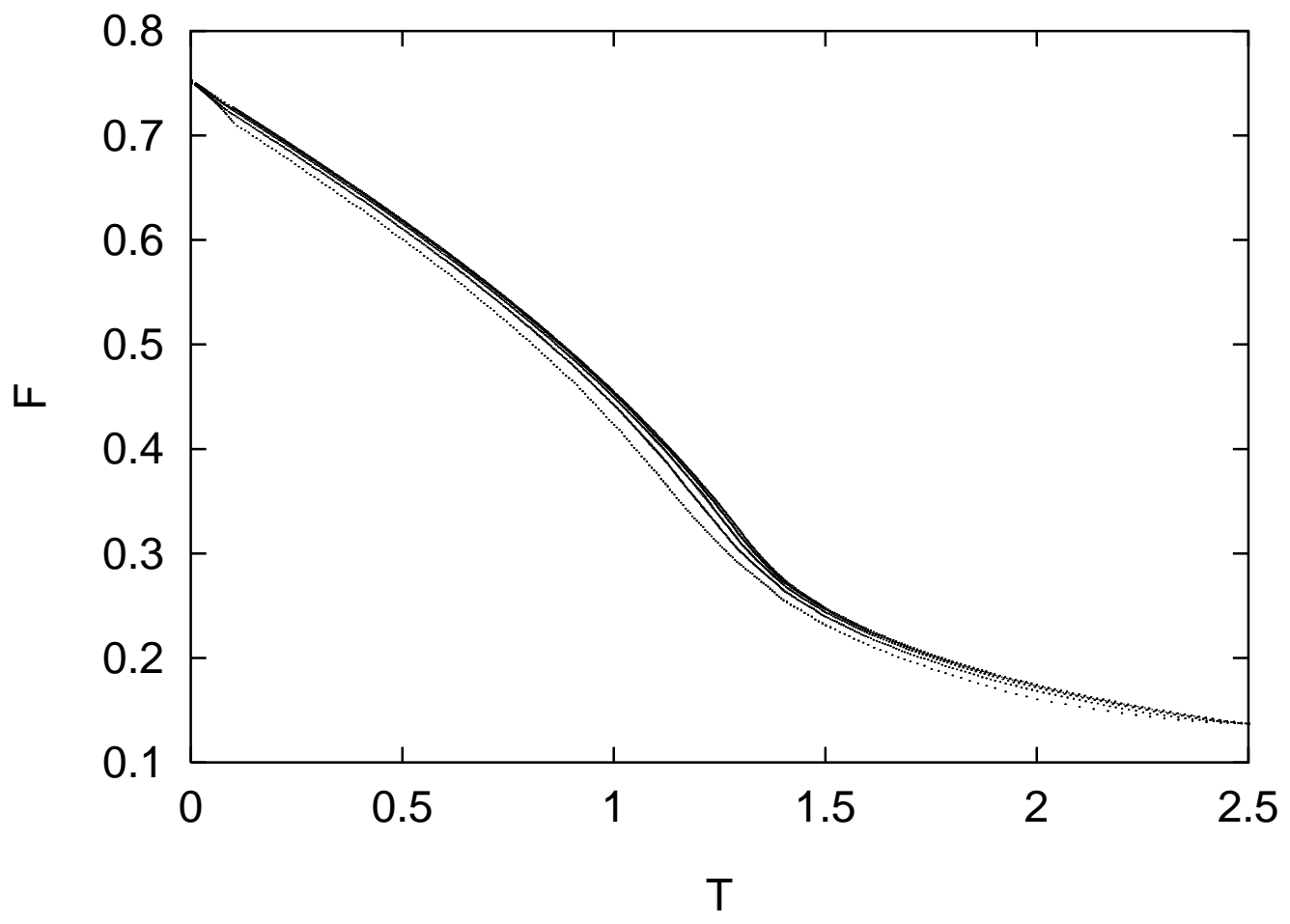

Figure 2: The spin functional $F$ as a function of the temperature $T$ for various cooling schedules. The curves correspond to $N_{s}=5200, N_{s}=2100, N_{s}=1100, N_{s}=550$, and $N_{s}=300$, respectively. 


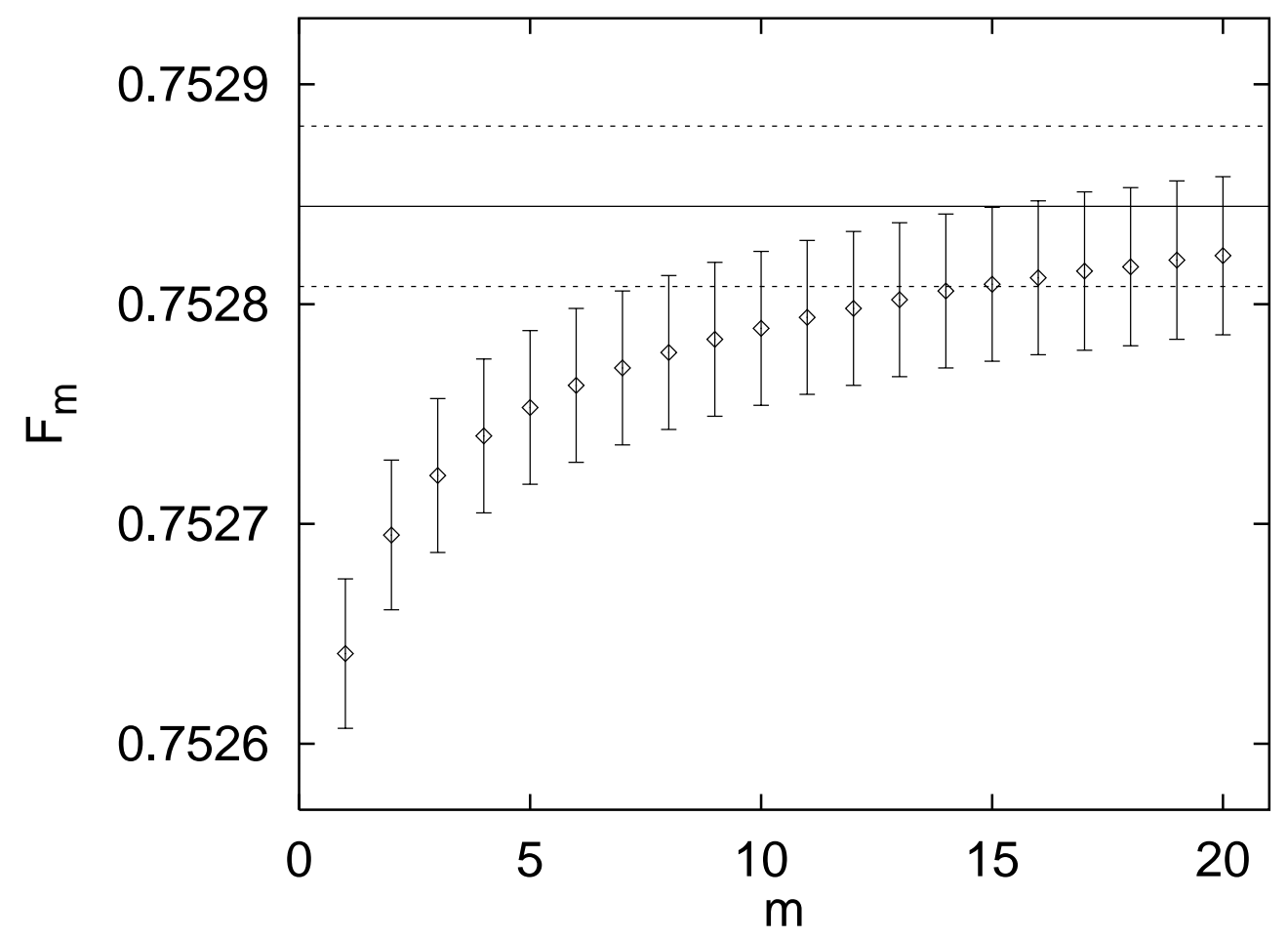

Figure 3: The maximized value of the spin functional $F$ as a function of the number of local maxima generated on each configuration. The solid line (with error band) represents our extrapolated value $(m \rightarrow \infty)$. 


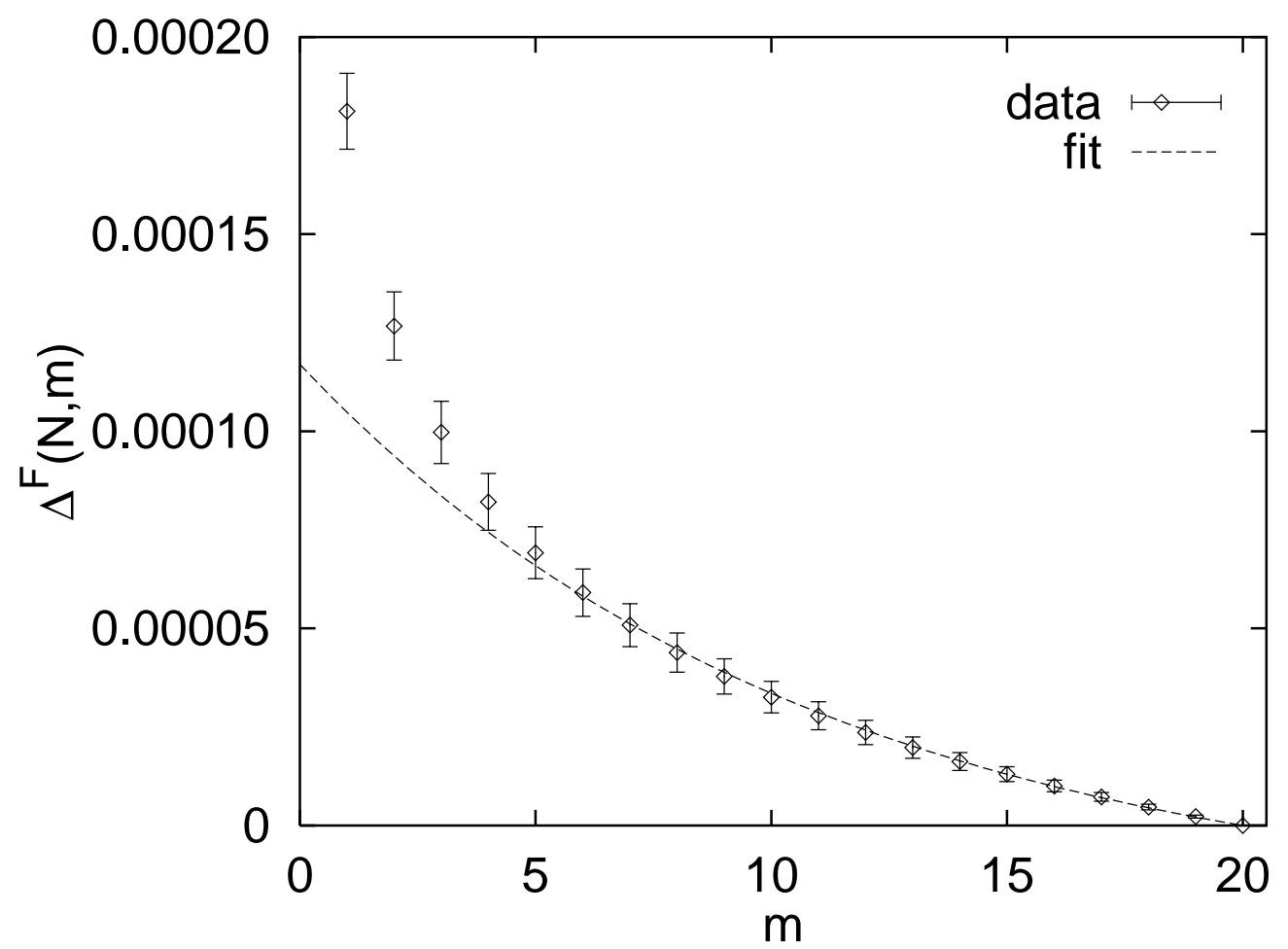

Figure 4: The differences, $\Delta^{F}(N, m)=F_{N}-F_{m}$ and an exponential fit. 


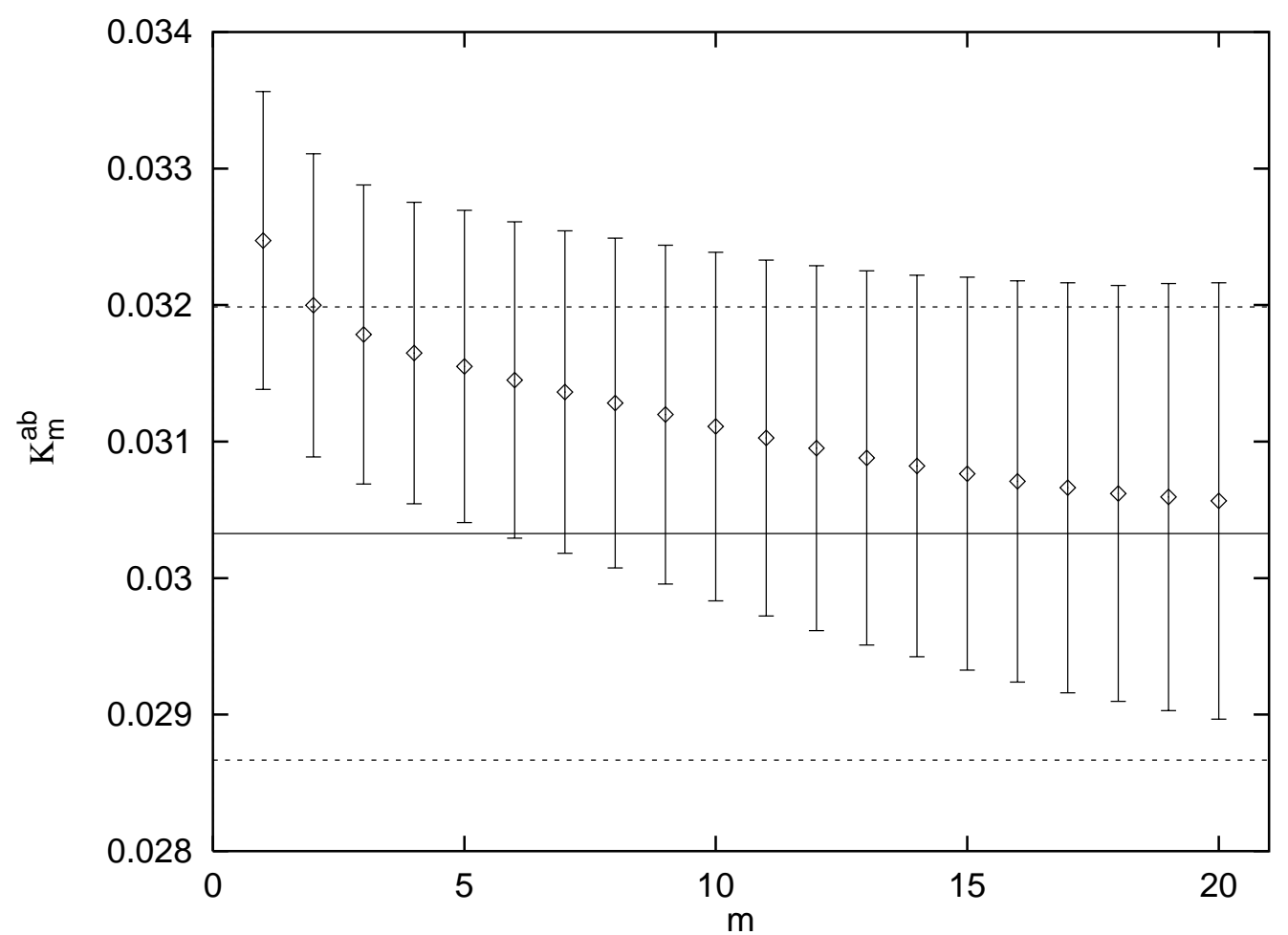

Figure 5: The abelian string tension versus the number of MA gauge copies $m$. The solid line (with error band) represents our extrapolated value $(m \rightarrow \infty)$. 


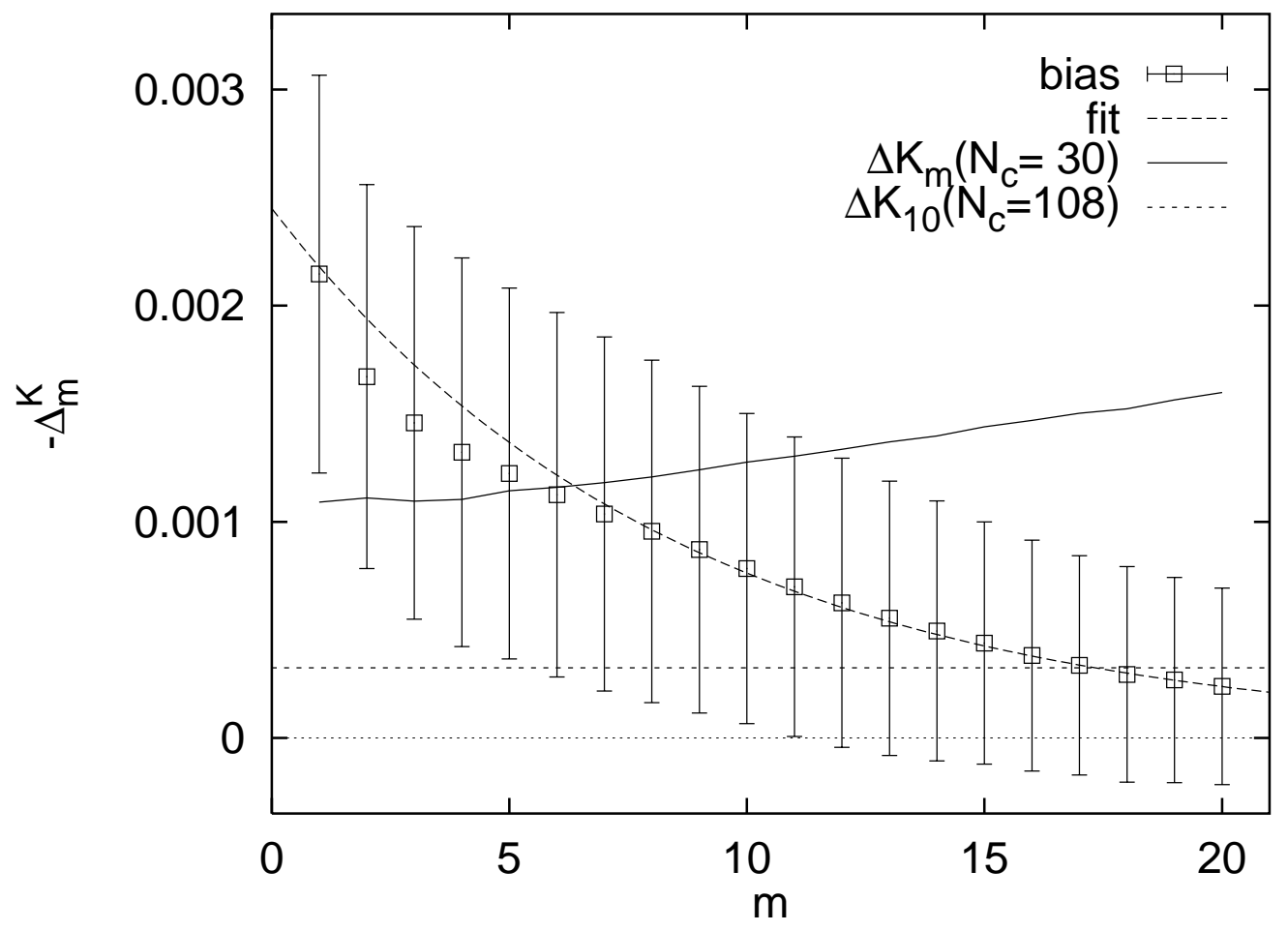

Figure 6: Differences between the extrapolated value $K_{\infty}^{a b}$ (from the exponential fit) and $K^{a b}$, obtained on a finite number of gauge copies, $m$. The solid line denotes the statistical uncertainty on $K^{a b}$ from 30 gauge configurations. The horizontal dashed line is the statistical uncertainty on 108 configurations with $m=10$. 


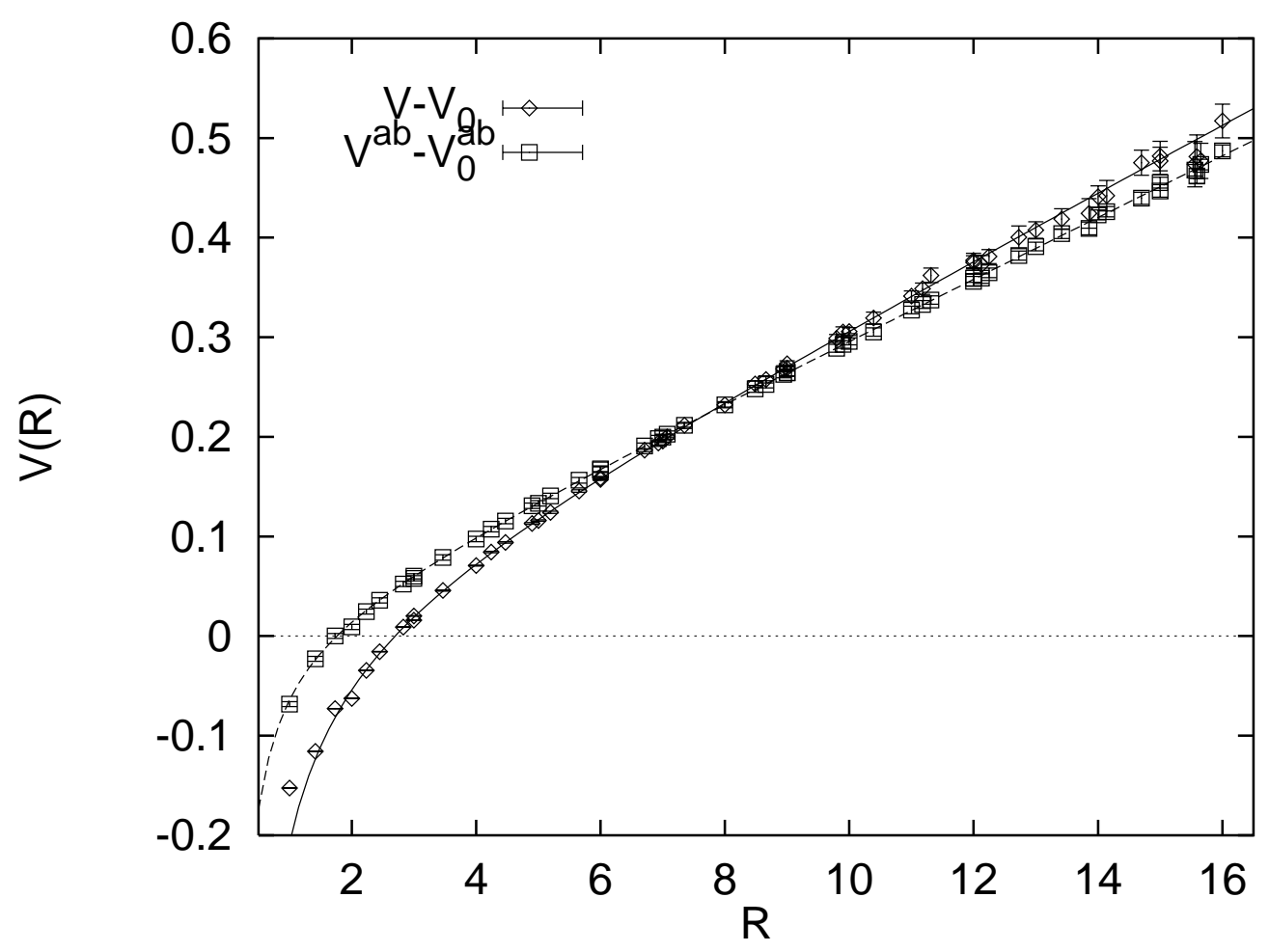

Figure 7: The abelian and non-abelian potentials, $V^{a b}$ and $V$. 


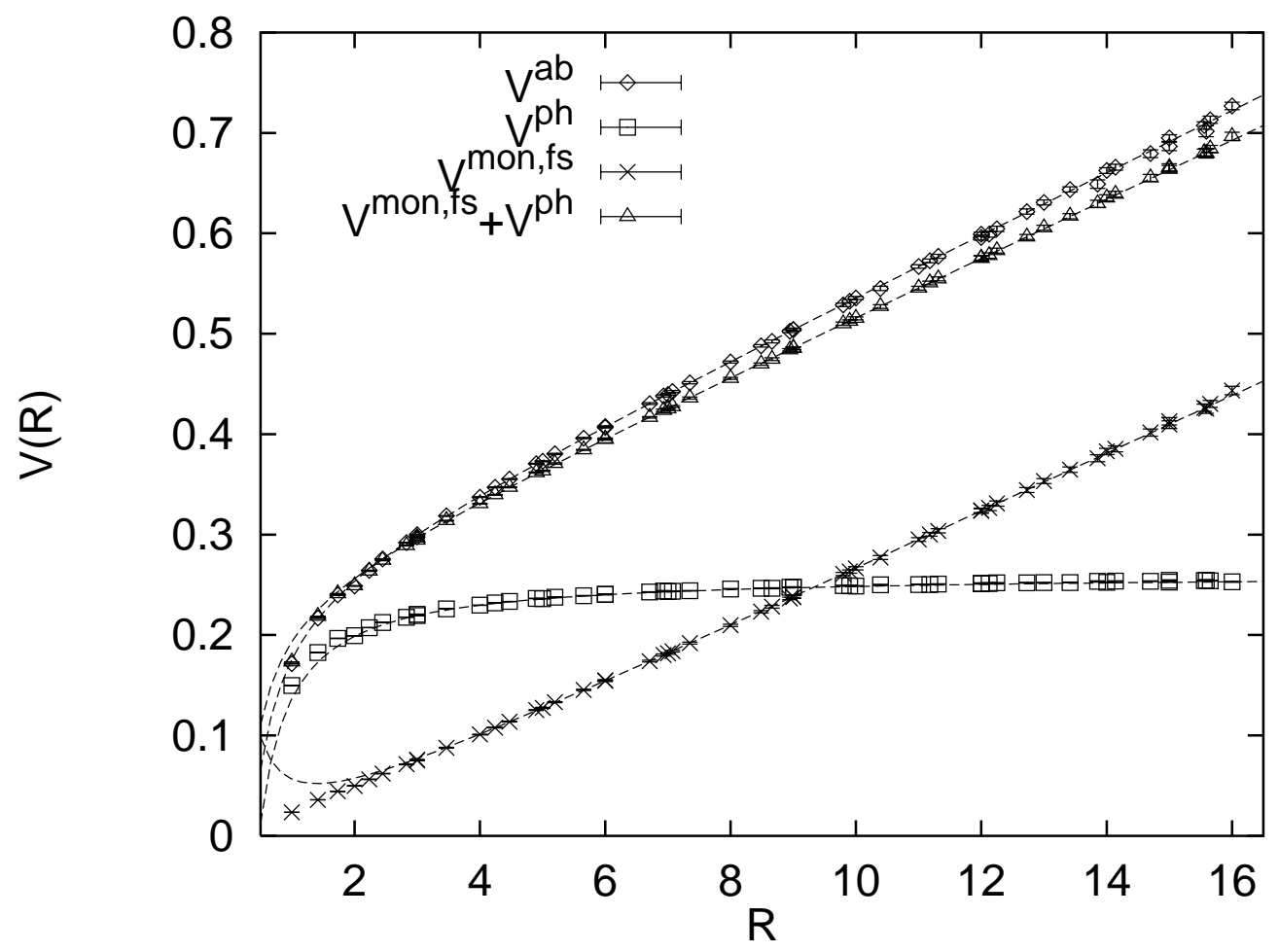

Figure 8: The abelian potential (diamonds) in comparison with the photon contribution (squares), the monopole (plus finite volume) contribution (crosses) and the sum of these two parts (triangles). Notice, that no (self energy) constants have been subtracted from any of the data sets. 


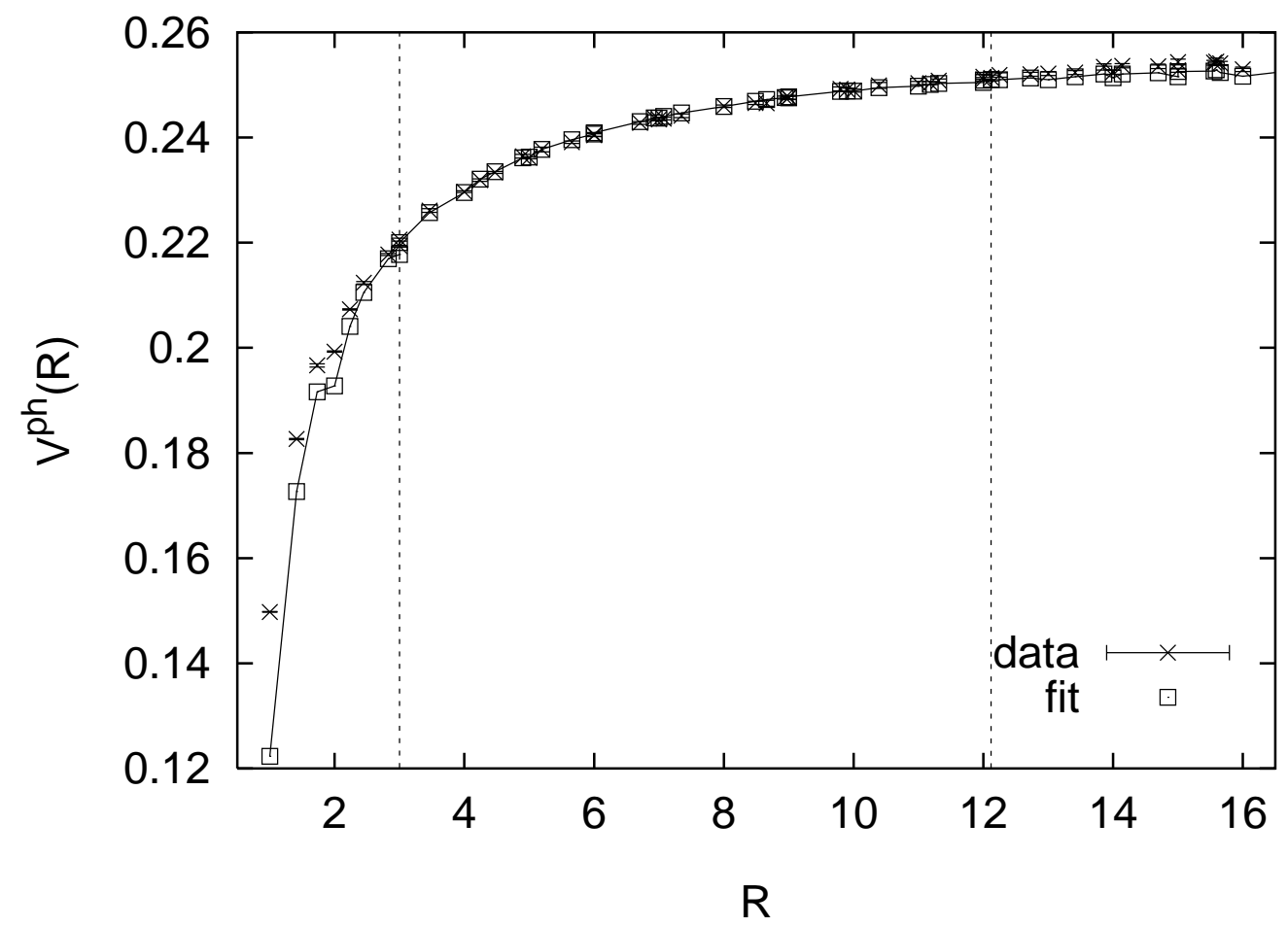

Figure 9: The photon contribution to the abelian potential. The dashed vertical lines indicate the fit range (solid curve and squares). 


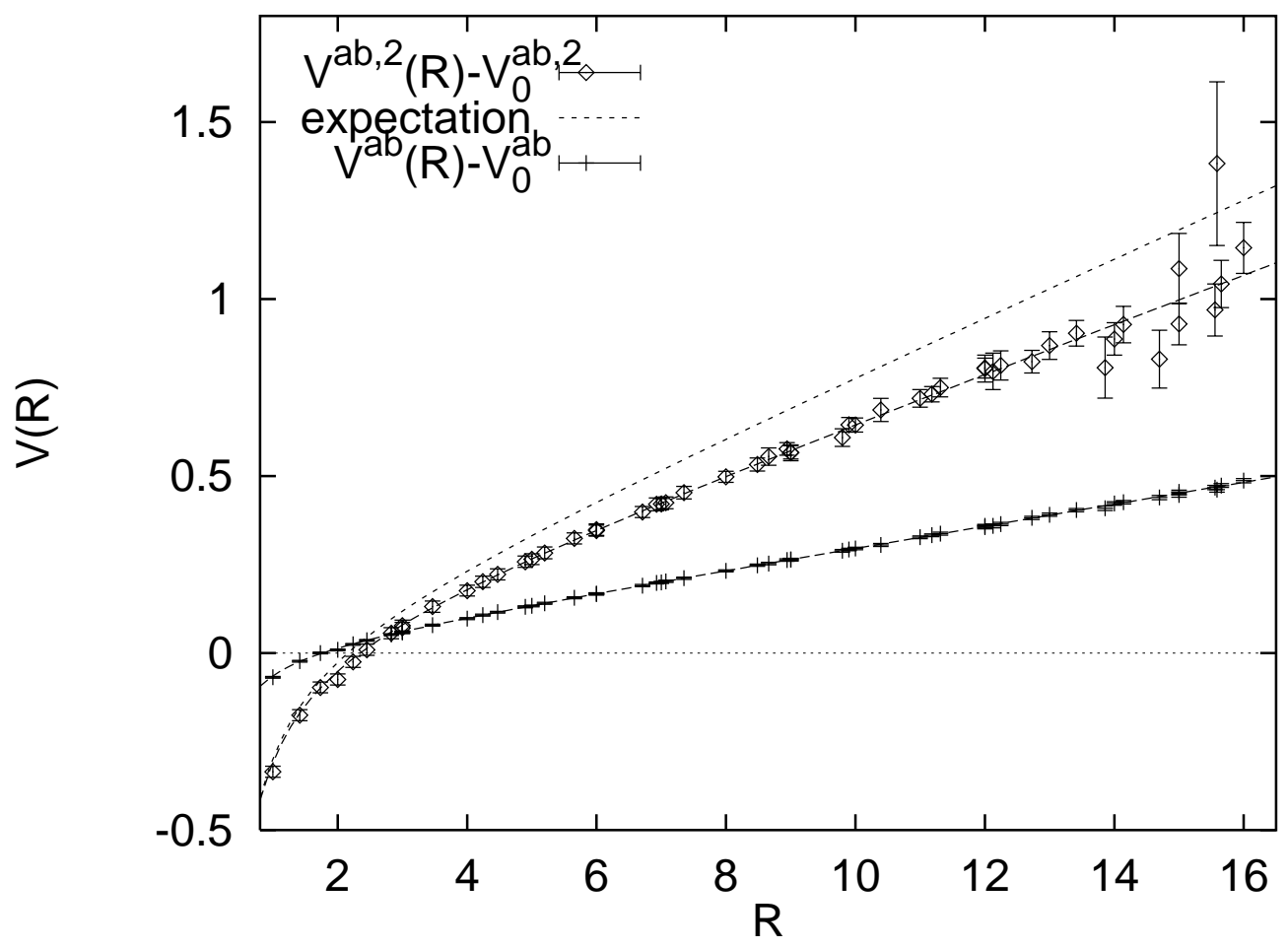

Figure 10: The static abelian charge two potential $V^{a b, 2}(R)$ in comparison to $V^{a b}(R)$. In addition, the expectation $V^{a b, 2}(R)-V_{0}^{a b, 2} \approx 8 / 3 K^{a b} R-4 e^{a b} / R$ is included (upmost dashed line). 